\title{
Distinct Sensorimotor Feedforward and Feedback Deficits Vary with Age in Individuals with Autism Spectrum disorder
}

\section{Kathryn E Unruh}

University of Kansas https://orcid.org/0000-0001-6745-5550

\section{Walker S McKinney}

University of Kansas

Kandace K Fleming

University of Kansas

John A Sweeney

University of Cincinnati

Matthew W Mosconi ( $\nabla$ mosconi@ku.edu )

University of Kansas https://orcid.org/0000-0002-1981-6777

\section{Research}

Keywords: autism spectrum disorder (ASD), typically developing (TD)

Posted Date: January 25th, 2021

DOI: https://doi.org/10.21203/rs.3.rs-152611/v1

License: (c) (1) This work is licensed under a Creative Commons Attribution 4.0 International License. Read Full License

Version of Record: A version of this preprint was published at Molecular Autism on July 10th, 2021. See the published version at https://doi.org/10.1186/s13229-021-00452-8. 
Distinct sensorimotor feedforward and feedback deficits vary with age in individuals with autism spectrum disorder

\author{
Kathryn E. Unruh \\ Life Span Institute, University of Kansas, Lawrence, KS \\ Kansas Center for Autism Research and Training (K-CART), University of Kansas, Lawrence, KS \\ Walker S. McKinney \\ Clinical Child Psychology Program, University of Kansas, Lawrence, KS \\ Kansas Center for Autism Research and Training (K-CART), University of Kansas, Lawrence, KS
}

Kandace K. Fleming

Life Span Institute, University of Kansas, Lawrence, KS

John A. Sweeney

Department of Psychiatry, University of Cincinnati, Cincinnati, $\mathrm{OH}$

Matthew W. Mosconi

Life Span Institute, University of Kansas, Lawrence, KS

Clinical Child Psychology Program, University of Kansas, Lawrence, KS

Kansas Center for Autism Research and Training (K-CART), University of Kansas, Lawrence, KS

Acknowledgements: This study was funded by grants NICHD U54 090216, NIMH R01 112734, and Kansas Center for Autism Research and Training (K-CART) Research Investment Council Strategic Initiative Grant. 


\begin{abstract}
Background: Sensorimotor issues are common in autism spectrum disorder (ASD), related to core symptoms, and predictive of worse functional outcomes. Deficits in rapid, feedforward processes executed prior to availability of sensory feedback, and continuous, feedback-guided motor behaviors each have been reported, but the degree to which these deficits are distinct or co-segregate in individuals is not well understood.
\end{abstract}

Methods: To characterize feedforward and feedback control of motor behavior in ASD, we examined saccadic eye movements (feedforward) and sustained precision gripping (feedback) in 109 individuals with ASD and 101 age-matched typically developing (TD) controls (range: 5-28 years). We measured latency and gain of saccades and error, variability, and regularity of precision grip. Linear mixed effects models were conducted to examine whether sensorimotor behavior varied according to diagnostic group, age, handedness, and sex.

Results: Individuals with ASD showed reduced accuracy of saccadic eye movements relative to controls, and their dysmetria was more severe at older ages. Individuals with ASD showed increased precision grip force variability relative to controls, especially at younger ages, while increased motor regularity was more pronounced in older individuals with ASD. Feedforward and feedback motor behaviors were strongly inter-related among controls, but not among individuals with ASD. Saccade dysmetria and increased force variability were associated with ASD symptom severity.

Limitations: Our age-related findings rely on cross-sectional data. Longitudinal studies of component motor skills and their associations with clinical outcomes are needed to clarify neurodevelopmental mechanisms of core and associated symptoms of ASD. Feedforward behavior was characterized in the oculomotor system using ballistic movements completed too rapidly to be guided by online feedback; however, future studies are needed to examine feedforward and feedback processes across both manual and oculomotor systems.

Conclusions: These findings suggest that separate neurodevelopmental mechanisms contribute to feedforward and feedback motor deficits in ASD, and that they are more manifest at different stages in life span development. Our results highlight the needs for more fine-grained approaches to parse separate motor impairments that often are considered as a unitary deficit in ASD, and to characterize variation in motor behaviors across development. 


\section{Introduction}

Sensorimotor deficits are common in autism spectrum disorder (ASD), emerge within the first years of life, often prior to social-communication issues (Bryson et al., 2007; Elberling et al., 2014) and are predictive of worse functional outcomes (Dziuk et al., 2007; Travers et al., 2017). While sensorimotor issues repeatedly have been demonstrated in ASD, the majority of existing studies have either approached sensorimotor behaviors as a unitary construct or interrogated component motor processes in isolation. Further, despite evidence that the severity of sensorimotor issues may vary considerably across development (Freitag et al., 2007; Luna et al., 2004), few studies have characterized age-dependent differences in select sensorimotor behaviors. As a result, knowledge of the specific motor control processes that are affected in ASD and understanding of their neurodevelopmental substrates remain limited. There is great need to define the nature of sensorimotor issues in ASD, test their variance across development, and determine the relationships among different sensorimotor behaviors in order to better clarify neurodevelopmental mechanisms contributing to sensorimotor disturbances in ASD that are related to clinical outcomes.

Individuals with ASD show a diverse range of sensorimotor issues, including increased spatial variability and temporal regularity of sustained precision gripping (Mosconi, Mohanty, et al., 2015; Wang et al., 2015), increased postural variability (Bojanek et al., 2020; Fournier et al., 2010; Wang et al., 2016), reduced coordination of complex lower (e.g., gait; Manicolo et al., 2019) and upper body movements (e.g., reaching; Glazebrook et al., 2006, 2009), and reduced accuracy and velocity of saccadic and smooth pursuit eye movements (Luna et al., 2007; Minshew et al., 1999; Schmitt et al., 2014; Sweeney et al., 2004). Increased variability of motor behavior in ASD suggests that feedback processes involved in integrating sensory information to reactively adjust the precision of ongoing motor output are deficient. Increased regularity of the time-dependent structure of motor output (i.e., reduced entropy) in ASD indicates a reduced ability to integrate the multiple feedback and feedforward processes involved in dynamically adjusting ongoing behavior. Reduced accuracy of saccades in ASD implicates feedforward processes that are guided by internal models that support output accuracy prior to sensory feedback being available based on afferent delays. Impairments in feedback and feedforward processes point to cerebellum, a region frequently implicated in the pathophysiology of ASD (Fatemi et al., 2012; Mosconi, Wang, et al., 2015) and showing atypical activation in individuals with ASD during simple motor tasks (Allen et al., 2004; Allen \& Courchesne, 2003; Unruh et al., 2019). These findings suggest dysfunction of multiple cerebellar processes including those for consolidation and planning of initial motor output and subsequent integration of 
motor and sensory feedback to optimize and stabilize continuous movement. The proposed studies aimed to determine the extent to which deficits in feedback and feedforward processes covary within individuals and vary across development in ASD.

We tested 109 individuals with ASD to characterize both feedback-guided precision gripping and saccadic eye movements under feedforward control. Feedback processes were characterized using a visuomotor task developed to target frontal- and parietal-cerebellar sensorimotor systems (Vaillancourt et al., 2003), while feedforward processes were studied using ballistic eye movements completed prior to online feedback adjustments. We aimed to determine whether sensorimotor abilities were similarly disrupted across motor control processes (feedback and feedforward) and how they are manifest over a wide age range (5-28 years). To determine the extent to which sensorimotor issues covary with core ASD symptoms as previously suggested (Dowell et al., 2009; Dziuk et al., 2007; Travers et al., 2013), we also examined associations with social-communication abnormalities and restricted, repetitive behaviors (RRBs). We predicted that individuals with ASD would show continuous precision gripping output that was less responsive to multisensory feedback (increased variability and regularity) implicating disruptions of feedback control processes. Further, consistent with our prior work (Mosconi, Mohanty, et al., 2015) we predicted that group differences in variability and regularity would be more pronounced when visual feedback was manipulated (i.e., amplified or degraded). We also predicted reduced accuracy of ballistic eye movements implicating disruption of feedforward control processes. Consistent with our prior small sample studies, it was hypothesized that increases in variability and regularity of continuous gripping and reductions in saccade accuracy in individuals with ASD would be more severe in younger individuals (Luna et al., 2007; Mosconi, Mohanty, et al., 2015; Wang et al., 2015). Based on prior findings from our group and others (Dowell et al., 2009; Mosconi, Mohanty, et al., 2015; Schmitt et al., 2014; Travers et al., 2013; Wang et al., 2015), we predicted that deficits in sensorimotor function would be associated with core social communication and repetitive behavior symptoms of ASD suggesting overlapping pathophysiological mechanisms.

\section{Methods}

\section{Participants}

109 individuals with ASD (20 female) and 101 typically developing (TD) controls (28 female) completed clinical testing and three sensorimotor tests, including two precision gripping tests in which force (Force test) or visual gain levels (Gain 
test) were varied, and a test of visually guided saccades (VGS test). Some individuals did not complete each test based on scheduling issues (Table 1). Individuals were studied at either the University of Illinois at Chicago (UIC) or the University of Texas Southwestern Medical Center (UTSW). Participant groups were similar in terms of sex ratio and handedness.

Participants with ASD were recruited through outpatient clinics and community advertisements. Participants completed one of three tests of general cognitive ability selected based on their age. At UIC, participants under 12 years of age completed the Differential Ability Scale, Second Edition (DAS), while participants $\geq 12$ years completed the Wechsler Abbreviated Scale of Intelligence (WASI). At UTSW, participants under 6 years of age completed the Wechsler Preschool and Primary Scales of Intelligence, Fourth Edition (WPPSI), while participants $\geq 6$ years completed the WASI.

\begin{tabular}{|c|c|c|c|c|c|c|c|}
\hline & & \multicolumn{2}{|c|}{ TOTAL } & \multicolumn{2}{|c|}{ UIC } & \multicolumn{2}{|c|}{ UTSW } \\
\hline & & Control & $\underline{\mathrm{ASD}}$ & Control & $\underline{\mathrm{ASD}}$ & Control & $\underline{\mathrm{ASD}}$ \\
\hline \multirow[t]{6}{*}{$\begin{array}{l}\text { Gripping } \\
\text { (Force test) }\end{array}$} & $\mathbf{N}$ & $74(20 \mathrm{~F})$ & $76(11 \mathrm{~F})$ & $41(10 \mathrm{~F})$ & $33(6 \mathrm{~F})$ & $33(10 \mathrm{~F})$ & $43(5 \mathrm{~F})$ \\
\hline & Age & $13.8(6.9)$ & $12.2(5.3)$ & $14.3(6.0)$ & $14.6(5.7)$ & $13.0(7.9)$ & $\begin{array}{l}10.3 \\
(4.0) \\
\end{array}$ \\
\hline & $\begin{array}{l}\text { Handedness } \\
(\mathrm{R} / \mathrm{L})\end{array}$ & $66 / 5$ & $61 / 14$ & $39 / 2$ & $30 / 3$ & $30 / 3$ & $32 / 11$ \\
\hline & Verbal IQ & $111(16)$ & $96(18)^{*}$ & $110(19)$ & $99(19)$ & $113(10)$ & $92(15)$ \\
\hline & $\begin{array}{l}\text { Nonverbal } \\
\text { IQ }\end{array}$ & 107 (15) & $100(18)^{*}$ & $105(14)$ & $99(18)$ & $108(15)$ & $100(17)$ \\
\hline & ADOS CSS & -- & 7.05 (1.87) & -- & $6.80(2.00)$ & -- & $\begin{array}{c}7.26 \\
(1.75)\end{array}$ \\
\hline \multirow[t]{6}{*}{$\begin{array}{l}\text { Gripping } \\
\text { (Gain test) }\end{array}$} & $\mathbf{N}$ & 59 (14) & $45(7 \mathrm{~F})$ & $40(10 \mathrm{~F})$ & $33(6 \mathrm{~F})$ & $19(5 \mathrm{~F})$ & $12(2 \mathrm{~F})$ \\
\hline & Age & $13.0(6.6)$ & $13.2(5.7)$ & $14.7(6.1)$ & $14.8(5.7)$ & $9.9(6.5)$ & $9.1(3.3)$ \\
\hline & $\begin{array}{l}\text { Handedness } \\
(\mathrm{R} / \mathrm{L})\end{array}$ & $50 / 5$ & $37 / 6$ & $37 / 3$ & $30 / 3$ & $16 / 3$ & $9 / 3$ \\
\hline & Verbal IQ & $111(17)$ & $98(19)^{*}$ & $110(19)$ & $100(20)$ & $113(12)$ & $86(14)$ \\
\hline & $\begin{array}{l}\text { Nonverbal } \\
\text { IQ }\end{array}$ & $106(16)$ & 100 (19) & $105(15)$ & $100(18)$ & $107(19)$ & $101(23)$ \\
\hline & ADOS CSS & -- & $6.81(1.85)$ & -- & $6.65(2.02)$ & -- & $\begin{array}{c}7.25 \\
(1.21) \\
\end{array}$ \\
\hline \multirow[t]{6}{*}{$\begin{array}{l}\text { Eye } \\
\text { movement } \\
\text { (VGS test) }\end{array}$} & $\mathbf{N}$ & $82(19 F)$ & $88(14 \mathrm{~F})$ & $55(13 \mathrm{~F})$ & $61(12 \mathrm{~F})$ & $27(7 \mathrm{~F})$ & $27(3 \mathrm{~F})$ \\
\hline & Age & $14.8(6.8)$ & $12.8(5.1)^{*}$ & $14.9(6.3)$ & $13.6(5.2)$ & $14.5(7.8)$ & $\begin{array}{l}11.1 \\
(4.2)\end{array}$ \\
\hline & $\begin{array}{l}\text { Handedness } \\
(\mathrm{R} / \mathrm{L})\end{array}$ & $75 / 4$ & $74 / 12$ & $50 / 3$ & $56 / 4$ & $25 / 1$ & $18 / 8$ \\
\hline & Verbal IQ & $110(13)$ & $97(18)^{*}$ & $109(16)$ & $99(18)$ & $114(11)$ & $92(18)$ \\
\hline & $\begin{array}{l}\text { Nonverbal } \\
\text { IQ }\end{array}$ & $106(13.5)$ & $101(18)^{*}$ & 104 (12.6) & $101(18)$ & $110(15)$ & 100 (19) \\
\hline & ADOS CSS & -- & $6.88(2.09)$ & -- & $6.84(2.21)$ & -- & $\begin{array}{c}7.00 \\
(1.78)\end{array}$ \\
\hline
\end{tabular}

Table 1. Participant Demographics. ASD, Autism spectrum disorder; F, Female; ADOS, Autism Diagnostic Observation Schedule; CSS, Calibrated Severity Score; VGS, Visually-guided saccade; Mean(SD). *group differences at $p<.05$. 
ASD diagnoses were confirmed using the Autism Diagnostic Inventory-Revised (ADI-R; Lord, Rutter, \& Le Couteur, 1994), the Autism Diagnostic Observation Schedule - Second Edition (ADOS; Lord et al., 2000), and based on expert clinical opinion using the Diagnostic and Statistical Manual of Mental Disorders (DSM) criteria. Participants assessed prior to 2013 were diagnosed according to DSM-IV TR, and all participants studied after publication of DSM-5 were diagnosed according to updated criteria. Participants with ASD were excluded for known genetic or metabolic disorders associated with ASD (e.g., fragile X syndrome, Tuberous sclerosis). Handedness was determined using self-report.

General exclusion criteria included self- or caregiver report of any history of substance dependence or abuse within the previous six months, history of non-febrile seizures or head trauma with loss of consciousness, complications during pregnancy, delivery, or perinatal period, or current use of medications known to interfere with sensorimotor behavior including stimulants, antipsychotics, anticonvulsants or benzodiazepines (Reilly et al., 2008). TD controls were excluded if they had a known lifetime history of psychiatric or significant medical disorder, had a family history of a major psychiatric disorder in their first-degree relatives, or a history of ASD in first or second-degree relatives. Participants refrained from caffeine, nicotine, and alcohol on the day of testing and over-the-counter drugs with sedating properties (e.g., cold medicine) within 12 hours of testing. Written informed consent was obtained from all participants, with assent and parental consent obtained for minors. Study procedures were approved by the local Institutional Review Boards.

\section{Precision grip testing}

Stimuli were presented on a $120 \mathrm{~cm}$ monitor with 1366 x 768 resolution and $120 \mathrm{~Hz}$ refresh rate. Participants were seated in a darkened room $53 \mathrm{~cm}$ from the display screen with their elbow at $90^{\circ}$ and arm in a relaxed position in a custom arm brace designed to keep the individual's arm steady throughout testing. Participants used their thumb and index finger to press against two opposing precision load cells (ELFF-B4-100N; Entran) $1.27 \mathrm{~cm}$ in diameter secured to a custom grip device attached to the arm brace (Figure 1a). A Coulbourn (V72-25) resistive bridge strain amplifier received analog signal from the load cells. Data were sampled at $120 \mathrm{~Hz}$ with a 16-bit analog-to-digital converter (DI-720; DATAQ Instruments) and converted to Newtons using a calibration factor derived from known weights before the study (Mosconi, Mohanty, et al., 2015). 


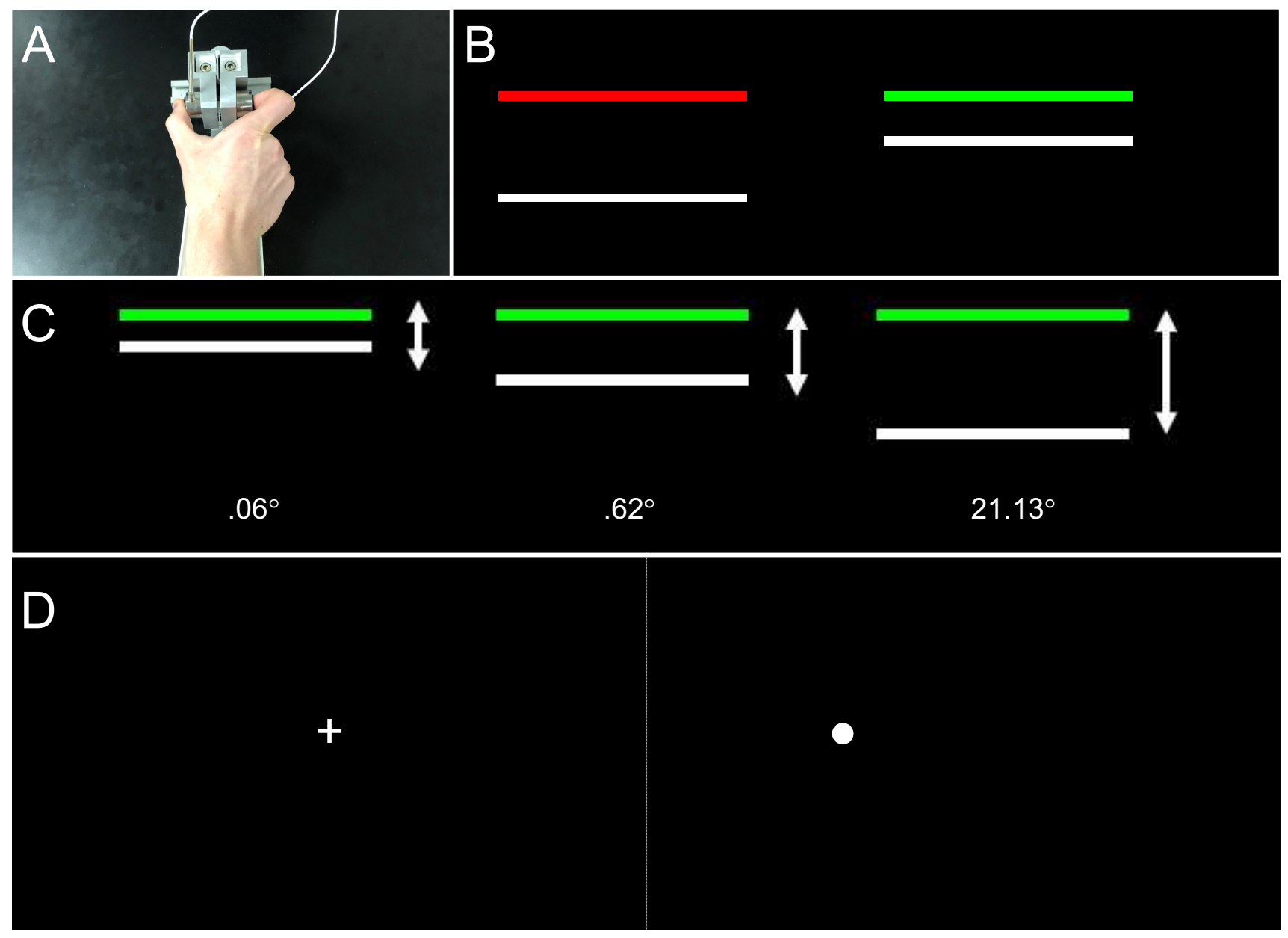

Figure 1. A. Precision grip load cell apparatus. B. Precision grip stimuli. Participants viewed a red TARGET bar and white FORCE bar. Once the TARGET bar turned green, participants pressed the load cells to bring the white FORCE bar up to the level of the green TARGET bar. C. Precision grip visual gain manipulation. The amount of visual feedback was either degraded (left: $0.06^{\circ}$ ) or amplified (rightmost: $21.13^{\circ}$ ). D. Visually guided saccade stimuli. Participants viewed crosshairs and made a saccade to the left $\left(-12^{\circ}\right.$; depicted) or right $\left(+12^{\circ}\right)$.

Before testing, each participant's maximum voluntary contraction (MVC) was calculated separately for each hand using the average of the maximum force output during three consecutive three second trials. During testing, participants viewed a horizontal white bar (FORCE) on a black screen that moved upward when force was applied to the load cells (Figure 1b). A static TARGET bar turned from red to green to indicate the beginning of each trial and participants were instructed to 1) press the load cells as quickly as possible when the TARGET bar turned green and 2) hold the FORCE bar steady at 
the level of the TARGET bar. At UIC, trials were $15 \mathrm{~s}$ and alternated with $15 \mathrm{~s}$ of rest. At UTSW, trials were $8 \mathrm{~s}$ and alternated with $8 \mathrm{~s}$ of rest.

Participants were administered two precision gripping tests (Figure 1). First, in order to determine the extent to which force load affected precision force control, participants were administered a Force Test in which they completed three trials with each hand at 15,45 , and $85 \%$ of their MVC. Visual angle was held constant at $0.62^{\circ}$. In order to determine the impact of changing the quality of visual feedback on precision force control, participants were administered a Gain Test in which the vertical distance the FORCE bar moved in response to changes in force output was varied (Figure 1c). Participants completed three trials with each hand at $0.06,0.62$, and $21.13^{\circ}$ of visual angle, consistent with our prior studies (Mosconi, Mohanty, et al., 2015; Wang et al., 2015). Precision grip test experiment order was counterbalanced across participants.

\section{Oculomotor testing}

Participants were tested in a dark room, seated $60 \mathrm{~cm}$ from a $102 \mathrm{~cm}$ anti-glare LCD monitor (resolution: 1920 x 1060) with head stabilized using a chin-rest to minimize movement (UIC refresh rate $=120 \mathrm{~Hz}$; UTSW refresh rate $=60 \mathrm{~Hz}$ ). At UIC, eye movements were recorded using infrared (IR) sclera-reflection sensors mounted on spectacle frames (Model 310, Applied Science Laboratories, Bedford, MA) using a 12-bit A/D converter (500 Hz; DI-720 from Dataq Instruments, Akron, $\mathrm{OH}$ ). Blinks were monitored using direct current electro-oculography (EOG; Grass Neurodata 12 Acquisition System; Astro-Med, Inc., West Warwick, RI). EOG electrodes were placed above and below the left eye and were linked to an AC-coupled bioamplifier. At UTSW, eye movements were recorded using an infrared, binocular camera-based eye tracking system (500 Hz; EyeLink II, SR Research Ltd., Canada). Across both sites, participants performed a nine-point calibration before each block of trials.

During the visually guided saccade test (Figure 1d), visual stimuli subtending $0.5^{\circ}$ of visual angle were presented in the horizonal plane at eye level. Following the presentation of a central fixation appearing for 1.5-2.5 s (varied randomly), a peripheral target was presented for $1.5 \mathrm{~s}$ at $\pm 12^{\circ}$. Fifteen trials were administered for each location (30 total trials); location order varied pseudo randomly. Participants were instructed to look to the target as quickly as possible. 


\section{Data processing}

\section{Precision grip data}

Force data were analyzed with a custom algorithm and scoring program developed previously by our group using MATLAB (MathWorks; Wang et al., 2015). The first two seconds and the last second of each force trace were excluded from analyses due to variability in the rate at which individuals reached the target force and terminated the trial (Robichaud et al., 2005). Trials for which participants produced fewer than 6 seconds of continuous force data were excluded from analyses. Trials also were excluded if the mean force exceeded twice the target force or was less than half of the target force. Force data were linearly detrended to account for systematic changes in the mean force over the duration of the trial. Data from each trial were visually inspected offline and scored blind to participant characteristics.

Error [(mean force of the time series / target force of the trial) - 1], force variability (coefficient of variation; CoV), and approximate entropy (ApEn) were examined. Positive error values represent "overshoot" of the target and negative error values represent target "undershoot". Force variability was defined as the standard deviation (SD) of the linearly detrended sustained force time series. To account for differences in variability due to individual differences in force output, the coefficient of variation $(\mathrm{CoV})$ was calculated by dividing the force SD by the mean force level for each trial. ApEn was calculated to examine the time-dependent structure of the force series (Pincus \& Goldberger, 1994; Slifkin \& Newell, 1999; Vaillancourt et al., 2001). ApEn values range from 0-2 and indicate the predictability of future values in a time series given a set of previous values, with lower numbers corresponding to more predictable data and higher numbers corresponding to more irregular, or complex, data.

\section{Oculomotor data}

Digital finite impulse response filters with non-linear transition bands were applied with a gradual transition band (from pass to no pass) between 20 and $65 \mathrm{~Hz}$ for velocity and position data, and between 30 and $65 \mathrm{~Hz}$ for acceleration data. Data from each trial were visually inspected offline and scored blind to participant characteristics. Trials were calibrated independently using fixation data from central and peripheral target locations. Each trial was manually calibrated by marking the stable center fixation prior to trial onset, and at the target location after the participant acquired the peripheral target. Trials were evaluated for signal drift and head movement and re-calibrated using within-trial data from fixation of targets of interest as we have done previously (Schmitt et al., 2014). Saccade onset and offset were marked where velocity 
exceeded or fell back below 30 deg per s, respectively. Trials with latencies $<70 \mathrm{~ms}$ were considered anticipatory and were not included in analyses. Trials were excluded if a blink occurred $100 \mathrm{~ms}$ prior to stimulus presentation or prior to the end of the primary saccade.

Saccade latency and gain were examined. Saccade latency was defined as the difference between peripheral target onset and saccade initiation. Saccade gain was defined as the ratio of the saccade amplitude to the target amplitude (Mosconi et al., 2010, 2013; Schmitt et al., 2014), with values below 1 indicating saccade hypometria and values greater than 1 indicating saccade hypermetria.

\section{Clinical measures}

In order to assess the severity of individuals' ASD symptoms, we examined the calibrated severity score (CSS) of the ADOS. The ADOS is a semi-structured assessment of social-communicative abnormalities and restricted, repetitive behaviors characteristic of ASD. The CSS is computed based on raw total percentiles that allow for comparison of symptom severity across ADOS modules selected based on age and language level (Gotham et al., 2009). Diagnostic algorithm scores from the ADI-R also were used to assess severity of social interaction and communication abnormalities and repetitive or stereotyped patterns of behavior. To examine subtypes of repetitive behavior, including repetitive sensorimotor behaviors, insistence on sameness, rituals, compulsions, and restricted interests, the Repetitive Behavior Scale-Revised (RBS-R; Lam \& Aman, 2007) also was used. Across all clinical measures, higher scores reflect greater symptom severity.

\section{Statistical Analyses}

To determine whether sensorimotor behavior differed according to diagnostic group, age, sex, or handedness, separate linear mixed effect analyses were conducted for each dependent variable of interest (Bates et al., 2015; Koller, 2016). Level one (within-subjects) predictors for the precision grip test included condition (force level or gain level) and hand (dominant vs. nondominant). Level one predictors for the eye movement test included target direction (left vs. right). Level two (between-subjects) predictors were the same for grip and eye movement tests and included age, sex, and diagnostic group. Location of data collection (UIC or UTSW) was included as a level two covariate of no interest. To limit the number of statistical analyses performed and maintain relatively parsimonious models, as is consistent with best- 
practice recommendations (Matuschek et al., 2017), initial models included only three-way interactions testing a priori hypotheses and their nested two-way interactions. To identify the best-fitting models, predictors were iteratively removed and model fit was compared between the previous and subsequent models using log likelihood ratio tests (Hox et al., 2017). Predictors that significantly improved model fit $(p<.05)$ were retained in the final model. Age was centered around the grand mean and categorical predictors were reference coded. Based on this scheme, model intercepts can be interpreted as follows. Force test: 15\% MVC performance for an average aged (13.1) male, healthy control, using their dominant hand; Gain test: $0.06^{\circ}$ visual angle performance for an average aged (13.1) male, healthy control, using their dominant hand; VGS: right target $\left(+12^{\circ}\right)$ performance for an average aged (13.7) male, healthy control. Main effects and interaction results are reported relative to these baseline reference values. Mixed effects modeling was conducted using the lme4 package (Bates et al., 2015) and reported model statistics were calculated using the lmerTest package (Kuznetsova et al., 2017) within R version 3.6.3

Linear regression models (using the base R stats package) were tested to examine the relationships between dependent variables within and across tasks and the strength of these relationships between groups. Spearman correlations (SPSS version 27) were computed to examine the relationships between motor variables that were different between groups and ADOS calibrated severity scores, ADI diagnostic algorithm scores, and RBS-R repetitive behavior subscale (stereotyped motor movements, self-injurious behavior, rituals, compulsions, insistence on sameness, and restricted interests) and total scores. These analyses included individuals with ASD only. For all correlation analyses, the Benjamini-Hochberg method was used to control for Type I error.

\section{Results}

\section{Precision grip testing: Maximum voluntary contraction}

MVC was reduced in individuals with ASD compared to controls $\left(F_{1,143}=2.62, p=.04\right)$, in the nondominant hand relative to the dominant hand $\left(F_{1,2106}=191.15, p<.001\right)$, in male participants compared to female participants $\left(F_{1,143}=4.65, p=\right.$ $.03)$, and improved with increased age $\left(F_{1,142}=58.73, p<.001\right)$.

\section{Precision grip testing: Force test}

Error 
Individuals showed relative target overshoot at low force and target undershoot at higher force levels. Error was greater at higher compared to lower target force levels $\left(F_{2,1833}=420.42, p<.001\right)$. Group differences in error varied as a function of force level (group x force: $F_{2,1835}=10.47, p<.001$; Figure 2). Follow-up analyses indicated that individuals with ASD demonstrated greater error than controls across all force levels, but that error at low force reflected target overshoot in $\operatorname{ASD}(t=-2.73, p<.001, d=.19)$, while error at medium $(t=3.42, p=.01, d=.26)$ and high force reflected target undershoot relative to controls $(t=3.70, p<.001, d=.32)$. Age-associations also varied as a function of target force level $\left(F_{2,1827}=44.11, p<.001\right)$. Increased age was associated with reduced error across all force levels, including less target overshoot at low force $\left(\beta=-0.01, t=-4.1, p<.001, R^{2}=.02\right)$ and less undershoot at medium and high force $(45 \% \mathrm{MVC}$ : $\beta=.01, t=2.49, p=.01, R^{2}=.01 ; 85 \%$ MVC: $\left.\beta=.01, t=8.43, p<.001, R^{2}=.12\right)$.

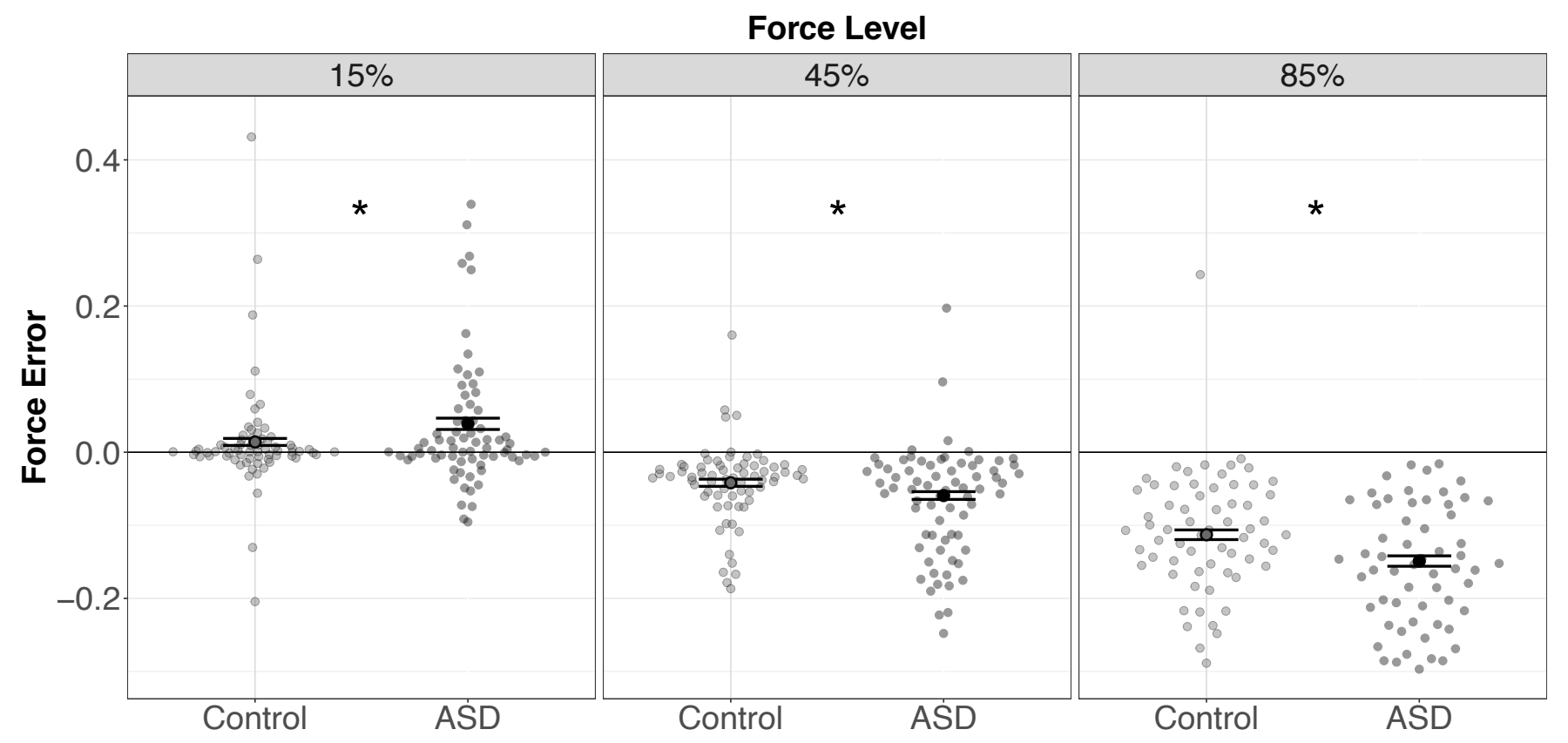

Figure 2. Force error was increased in ASD participants relative to TD controls across all levels of force. Compared to controls, individuals with ASD demonstrated target overshoot at 15\% and target undershoot at 45 and $85 \%$ MVC. Error bars reflect standard error of the mean. ${ }^{*} p<.05$.

\section{Coefficient of Variation}

$\mathrm{CoV}$ was greater at higher compared to lower target force levels $\left(F_{2,1963}=227.11, p<.001\right)$. Group differences in $\mathrm{CoV}$ varied as a function of age and target force level (age x group x MVC: $F_{2,1964}=6.59, p=.01$; Figure 3). Follow-up simple 
regression analyses identified a stronger inverse association between age and $\mathrm{CoV}$ in individuals with ASD relative to controls at low force only $\left(\beta=-0.03, t=-4.90, p<.001, R^{2}=.22\right)$, whereas age-associations with CoV were similar for individuals with ASD and controls at medium and high force levels.

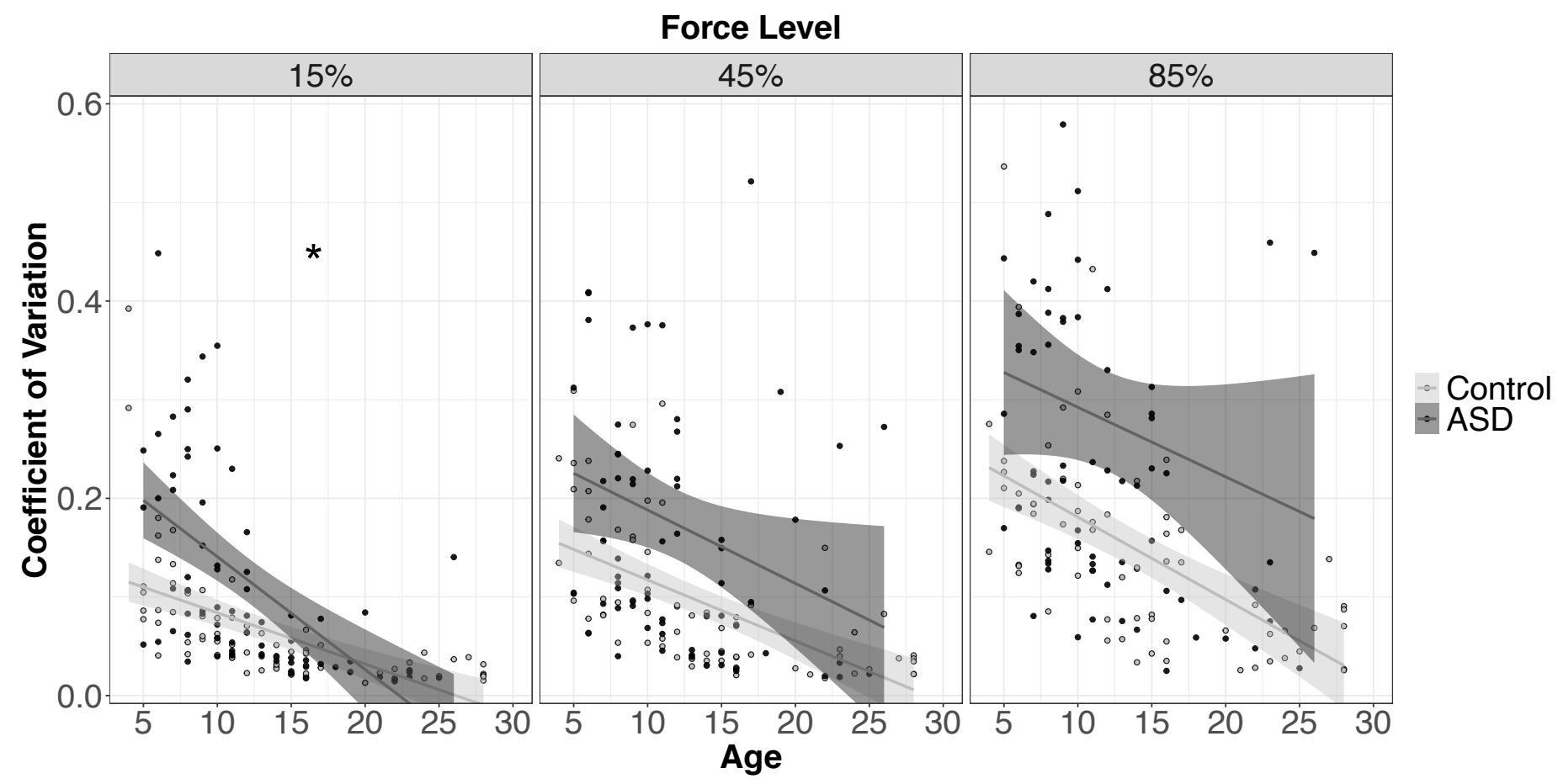

Figure 3. Force variability was increased in ASD participants relative to TD controls. Elevations in force variability were more severe in younger individuals with ASD relative to older individuals with ASD at 15\% MVC. Error bars reflect standard error of the mean. *slopes differ between groups.

\section{Approximate Entropy}

ApEn was reduced at medium and high force levels compared to low force $\left(F_{2,1993}=223.54, p<.001\right)$. Differences in ApEn between individuals with ASD and controls varied as a function of age and force level (age x group x MVC: $F_{2,1989}$ $=6.28, p=.01$; Figure 4). Follow-up regression analyses indicated the age $\mathrm{x}$ group $\mathrm{x}$ force level interaction reflected a weaker association between age and ApEn in individuals with ASD relative to controls at medium $(\beta=-0.03, t=-3.63, p<$ $\left..001, R^{2}=.24\right)$ and high force $\left(\beta=-0.04, t=-3.77, p<.001, R^{2}=.15\right)$. Group differences also varied as a function of sex and force level (sex x group x MVC: $F_{2,1993}=3.64, p=.03$; Supplementary Figure 1). ApEn was reduced in males with ASD compared to male control participants across all force levels $(15 \%: t=3.59, p<.001, d=.58 ; 45 \%: t=5.18, p<$ 
$.001, d=.84 ; 85 \%: t=2.64, p=.01, d=.43$ ), while females with ASD showed reduced ApEn compared to female control participants only at medium $(t=3.25, p=.01, d=1.22)$ and high force $(t=5.73, p<.001, d=2.15)$.

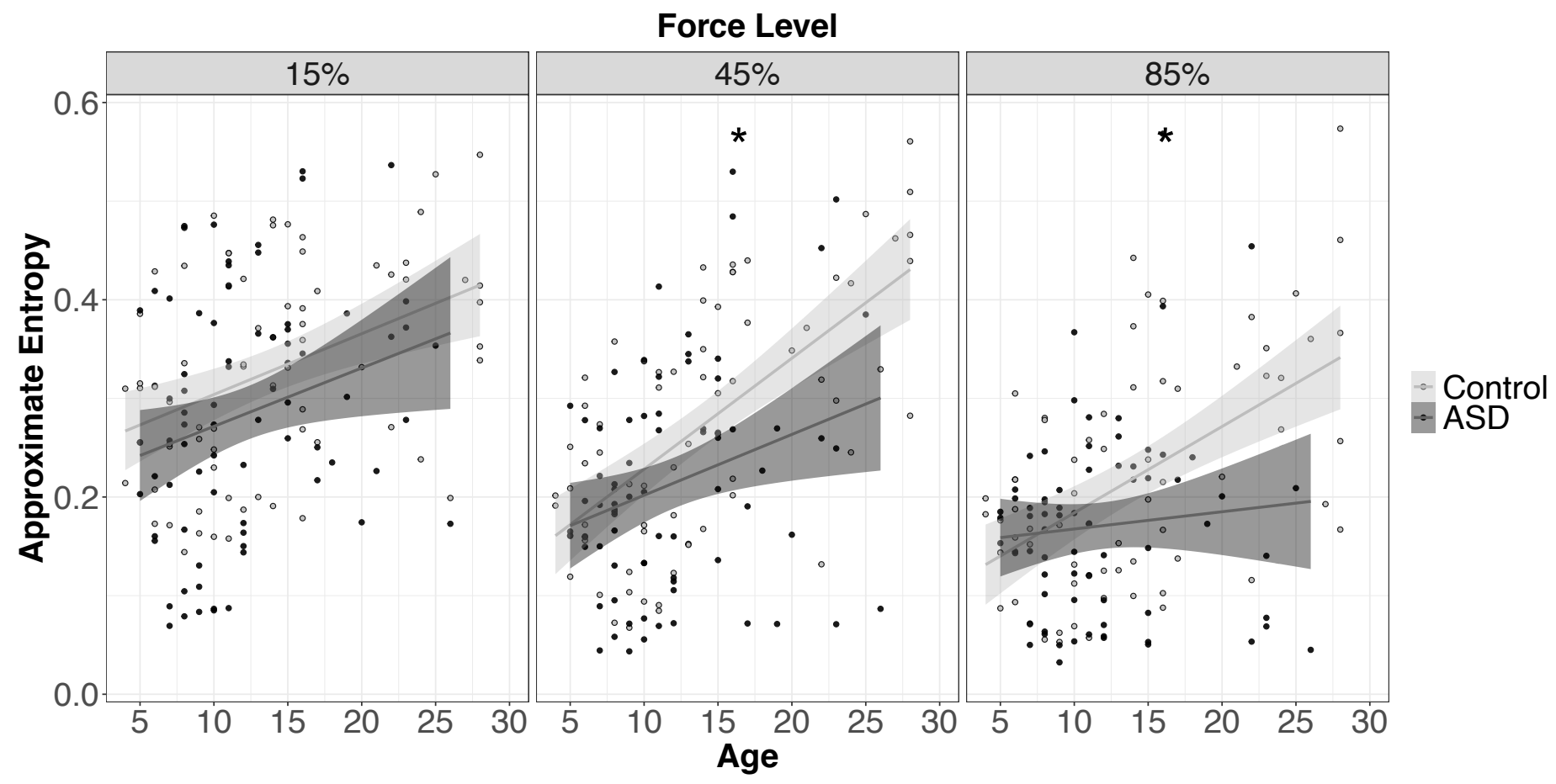

Figure 4. Approximate entropy is reduced in individuals with ASD compared to controls across all force levels. At 45 and 85\% MVC, individuals with ASD show reduced age-associated increases in ApEn compared to controls. Error bars reflect standard error of the mean. *slopes differ between groups.

\section{Gain Test}

Error

Force error was lower at medium gain relative to low and high gain $\left(F_{2,1427}=35.87, p<.001\right)$. Group differences in error varied as a function of hand (group $\mathrm{x}$ hand: $F_{1,1437}=10.06, p=.01$; Figure 5). Force error was similar between groups during nondominant hand gripping $(t=-0.05, p=.96)$, but individuals with ASD showed greater force overshoot than controls during dominant hand gripping $(t=-4.63, p<.001, d=.33)$. 


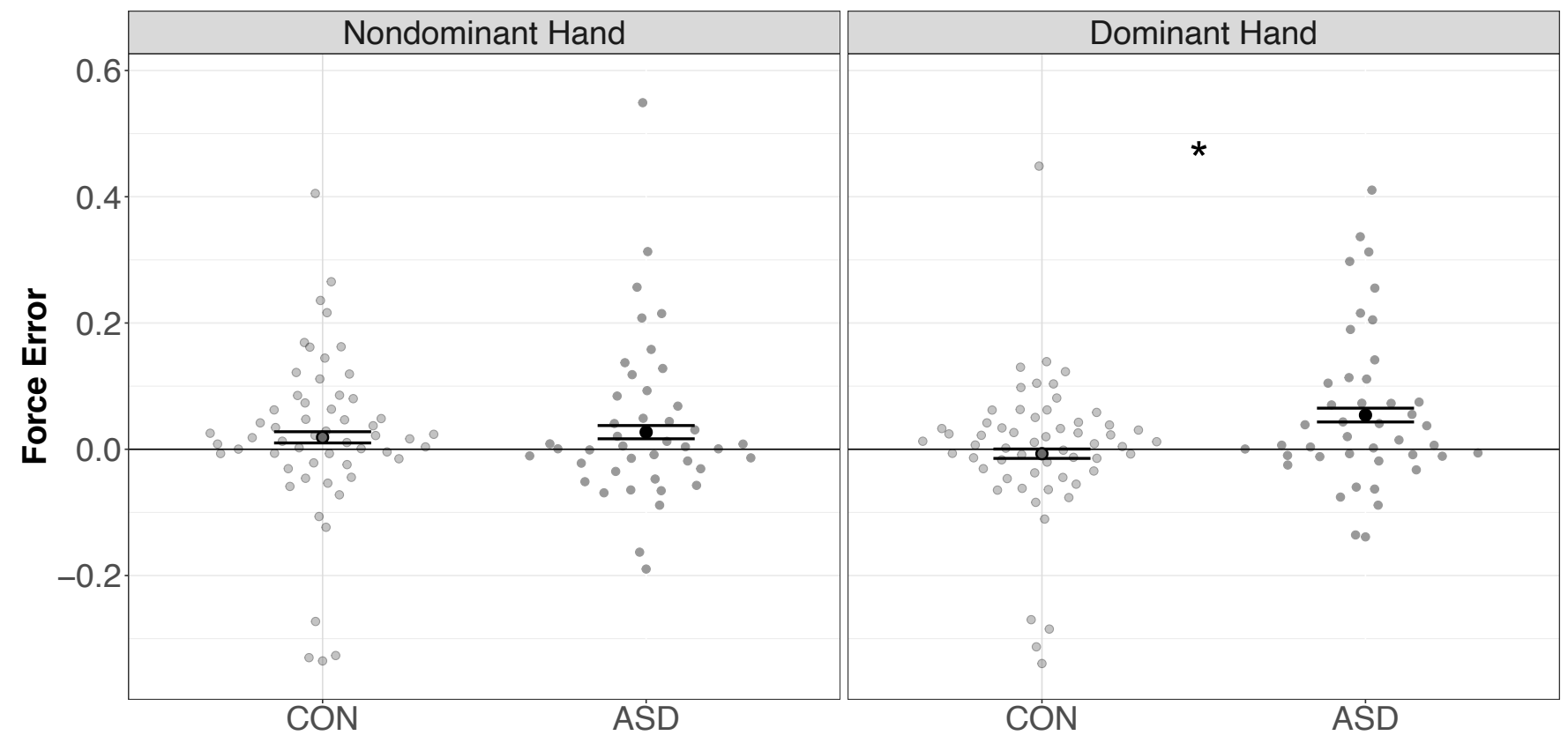

Figure 5. Force error was increased in ASD participants relative to TD controls when using the dominant hand. Negative values indicate target undershoot and positive values indicate target overshoot. Error bars reflect standard error of the mean. $* p<.05$.

\section{Coefficient of Variation}

$\mathrm{CoV}$ was elevated at low and high compared to medium gain $\left(F_{2,1450}=59.93, p<.001\right)$. Group differences in CoV varied as a function of age and gain level (age $\mathrm{x}$ group $\mathrm{x}$ visual angle: $F_{2,1450}=5.87, p=.01$; Figure 6 ). Follow-up regression analyses identified a stronger inverse association between age and $\mathrm{CoV}$ in individuals with ASD relative to controls at low $\left(\beta=-.03, t=-3.66, p<.001, R^{2}=.30\right)$ and medium gain $\left(\beta=-.02, t=-3.02, p=.01, R^{2}=.26\right)$, whereas age-associations with $\mathrm{CoV}$ were similar for individuals with ASD and controls at high gain. 


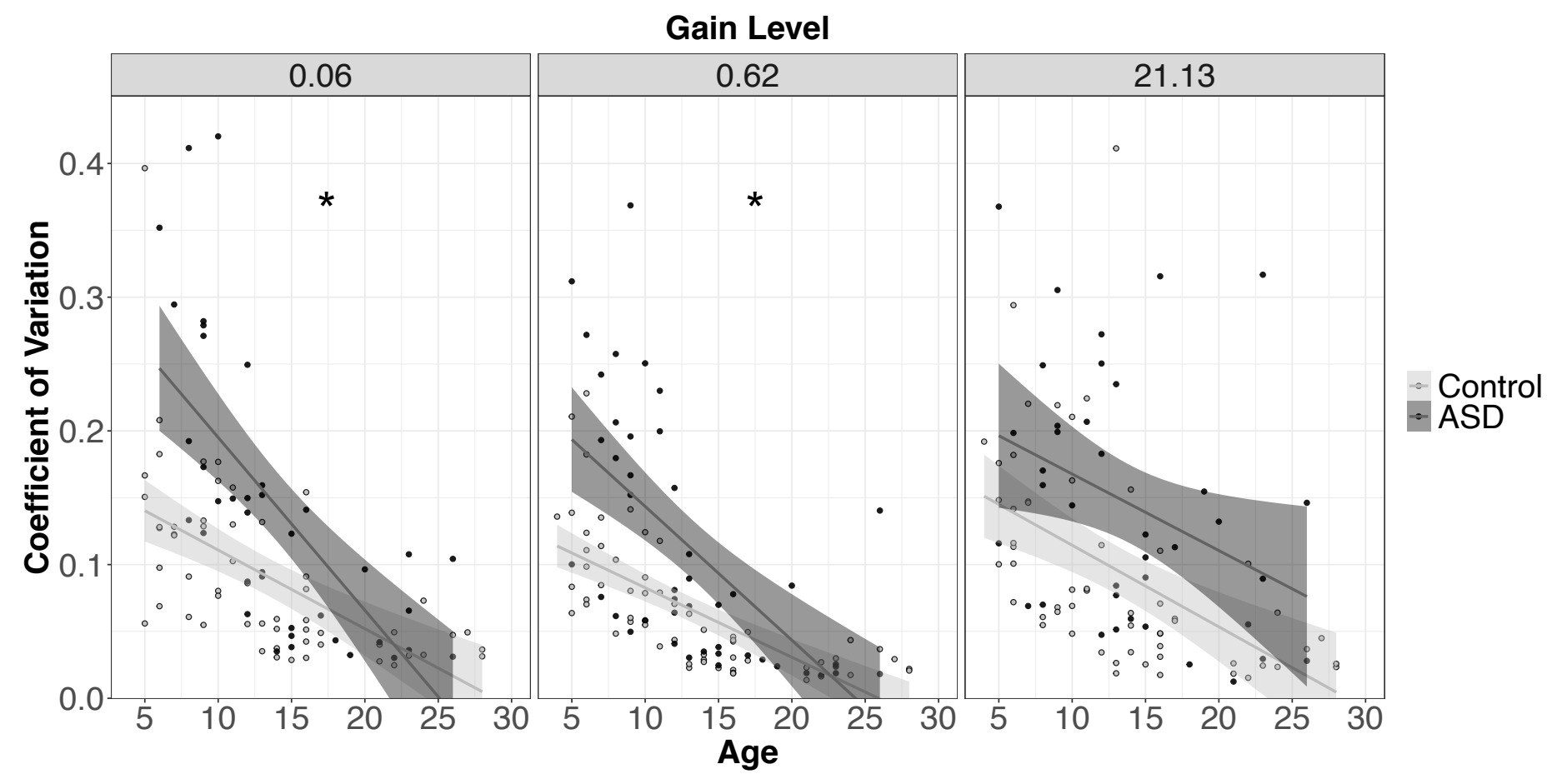

Figure 6. Variability of force output varies as a function of age and amount of visual feedback. Individuals with ASD demonstrated greater age-associated reductions in force variability (coefficient of variation) than control participants at low and medium gain. Age-related reductions in force variability were similar in ASD and controls at high gain, but overall variability was increased in ASD participants. Error bars reflect standard error of the mean. *slopes differ between groups.

\section{Approximate Entropy}

ApEn was reduced at high and low gain levels compared to medium gain $\left(F_{2,1399}=52.27, p<.001\right)$. Group differences in ApEn varied as a function of age and hand (age x group $\mathrm{x}$ hand: $F_{1,1405}=5.99, p=.01$; Supplementary Figure 2). Followup regression analyses indicated that increased age was less strongly associated with increased ApEn in individuals with ASD than controls for the non-dominant $\left(\beta=-.02, t=-2.03, p=.04, R^{2}=.14\right)$, but not the dominant hand $(\beta=.00, t=.94$, $p=.33)$.

\section{Visually-Guided Saccade Test}

\section{Latency}


Group differences in saccade latency varied as a function of target direction (group x target: $F_{1,4220}=4.23, p=.02$;

Supplementary Figure 3a) and sex $\left(F_{1,165}=4.20, p=.04\right.$; Supplementary Figure $\left.3 b\right)$. Saccade latencies were reduced in individuals with ASD relative to controls for rightward $(t=3.58, p<.001, d=.11)$ but not leftward targets $(t=0.51, p=$ $.61)$. Males with ASD showed reduced saccade latencies relative to control males $(t=5.05, p<.001, d=.17)$ while females with ASD showed increased saccade latencies relative to control females $(t=-3.02, p<.001, d=.19)$. Increased age was associated with faster saccade latencies across participants $\left(\beta=-10.96, t=-12.91, p<.001, R^{2}=.04\right)$.

\section{Gain}

Group differences in saccade gain varied as a function of age (group x age: $F_{2,166}=3.76, p=.02$; Figure 7 ). Follow-up simple regression analyses indicated increased age was associated with more accurate saccade gain in controls $(\beta=0.01, t$ $\left.=3.69, p<.001, R^{2}=.01\right)$ and more severe hypometria in $\operatorname{ASD}\left(\beta=-0.01, t=-3.55, p<.001, R^{2}=.01\right)$.

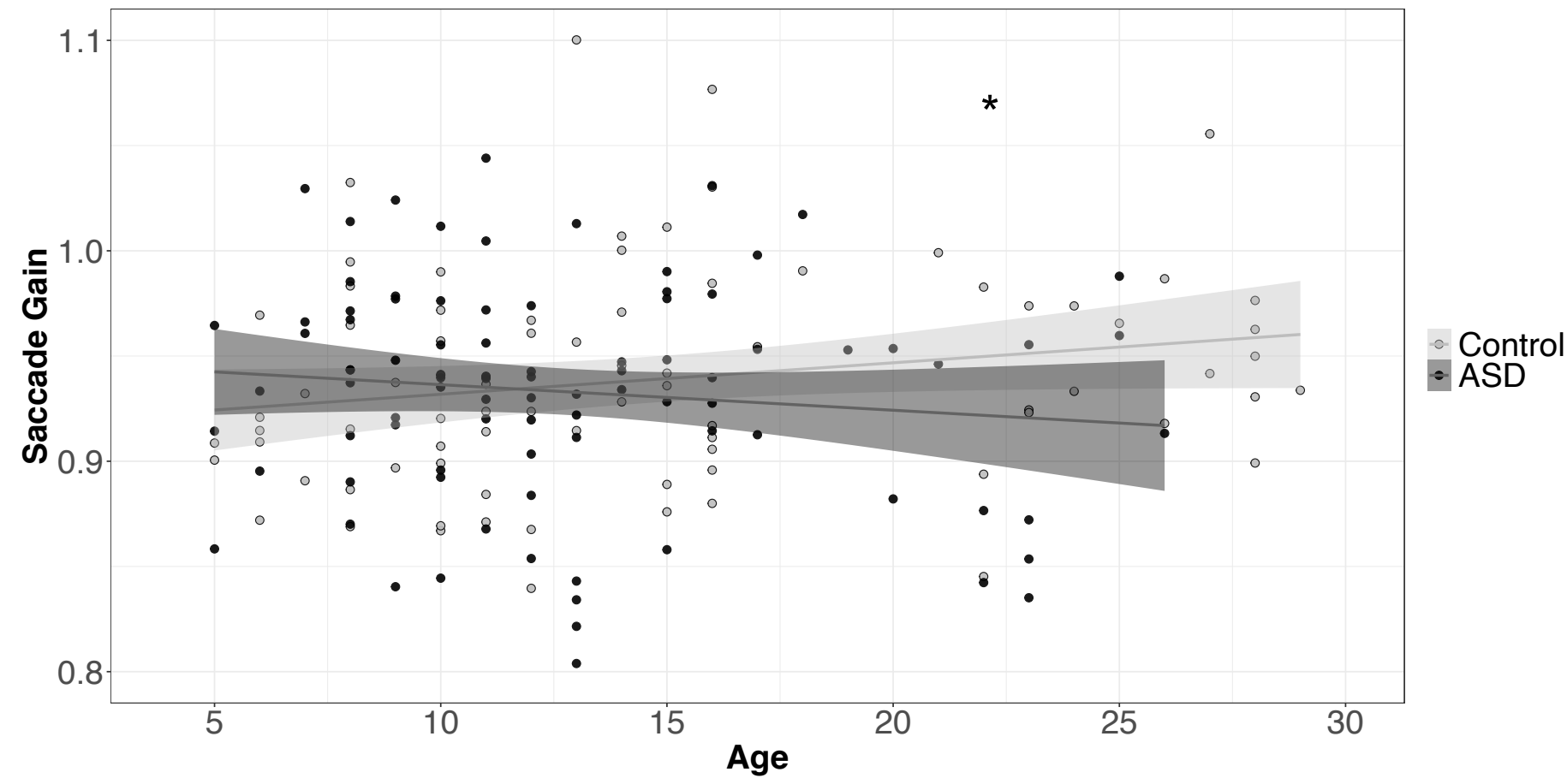

Figure 7. Increased age was associated with increased saccade accuracy in controls and with more severe hypometria in individuals with ASD. Error bars reflect standard error of the mean. *slopes differ between groups.

\section{Inter-Correlations of Sensorimotor Behaviors}


ApEn and CoV were negatively associated, but the strength of these associations varied across group and force level. At low force, ApEn and $\mathrm{CoV}$ were similarly associated across groups $\left(\beta=-.59, t=-8.70, p<.001 ; R^{2}=.34\right)$. At medium force, the association between ApEn and CoV was stronger in ASD compared to control participants (group x 45\% MVC: $\beta=.57, t=2.48, p=.01, R^{2}=.83$ ), while at high force, this association was stronger in controls compared to participants with ASD (group x 85\% MVC: $\beta=.63, t=2.50, p=.01, R^{2}=.34$ ). ApEn was associated with force undershoot at medium and high force (45\% MVC: $\beta=-.48, t=6.35, p<.001, R^{2}=.23 ; 85 \%$ MVC: $\beta=-.56, t=-7.67, p<.001, R^{2}=$ .31) but not at low force. CoV was associated with force overshoot and the strength of this association was stronger in controls compared to participants with ASD (group x 15\% MVC: $\beta=-.83, t=2.65, p=.01, R^{2}=.05$; group x 45\% MVC: $\beta=.48, t=2.31, p=.02, R^{2}=.52$; group x 85\% MVC: $\left.\beta=.42, t=3.10, p=.01, R^{2}=.58\right)$.

\section{Gain task relationships}

$\mathrm{ApEn}$ and $\mathrm{CoV}$ were negatively associated across all gain levels $\left(0.06\right.$ degrees: $\beta=-.62, t=-7.45, p<.001, R^{2}=.37 ; 0.62$ degrees: $\beta=-.70, t=-9.71, p<.001, R^{2}=.49 ; 21.13$ degrees: $\left.\beta=-.73, t=-10.10, p<.001, R^{2}=.54\right)$. The relationship between ApEn and error varied as a function of gain level. At low gain, ApEn and error were not associated. At medium gain, ApEn was associated with force overshoot $\left(\beta=.21, t=2.10, p=.04, R^{2}=.10\right)$, and at high gain, ApEn was associated with force undershoot in both groups $\left(\beta=-.31, t=-3.02, p=.01, R^{2}=.12\right)$. The relationship between CoV and error varied as a function of gain level and group. At low gain, CoV was associated with force undershoot in ASD, but not in controls (group x 0.06 degrees: $\beta=-1.05, t=-3.29, p=.01, R^{2}=.38$ ). At medium gain, CoV was associated with force undershoot in both groups $\left(\beta=-.35, t=-3.62, p<.001, R^{2}=.11\right)$, and at high gain, $\mathrm{CoV}$ was associated with force overshoot in both groups $\left(\beta=.27, t=2.57, p=.01, R^{2}=.08\right)$.

\section{Visually-guided saccade task relationships}

Saccade latency and gain were positively associated in both groups $\left(\beta=.19, t=2.53, p=.01, R^{2}=.04\right)$. 
Increased saccade gain was associated with reduced manual motor $\mathrm{CoV}$ in control participants, but not in participants with ASD, across several task conditions (group x 15\% MVC: $\beta=.30, t=3.25, p<.001, R^{2}=.10$; group x 0.62 degrees: $(\beta=$ $.28, t=2.46, p=.02, R^{2}=.15$; group $\mathrm{x} 21.13$ degrees: $\beta=.35, t=3.05, p=.01, R^{2}=.11$, Figure 8 ). Reduced saccade latency was associated with increased ApEn in controls, but not in individuals with ASD, across several task conditions (group x 45\% MVC: $\beta=-.26, t=-2.93, p=.01, R^{2}=.08$; group x 0.62 degrees: $\beta=-.35, t=-3.10, p=.01, R^{2}=.05$; group x 21.13 degrees: $\left.\beta=-.38, t=-3.28, p=.01, R^{2}=.08\right)$.

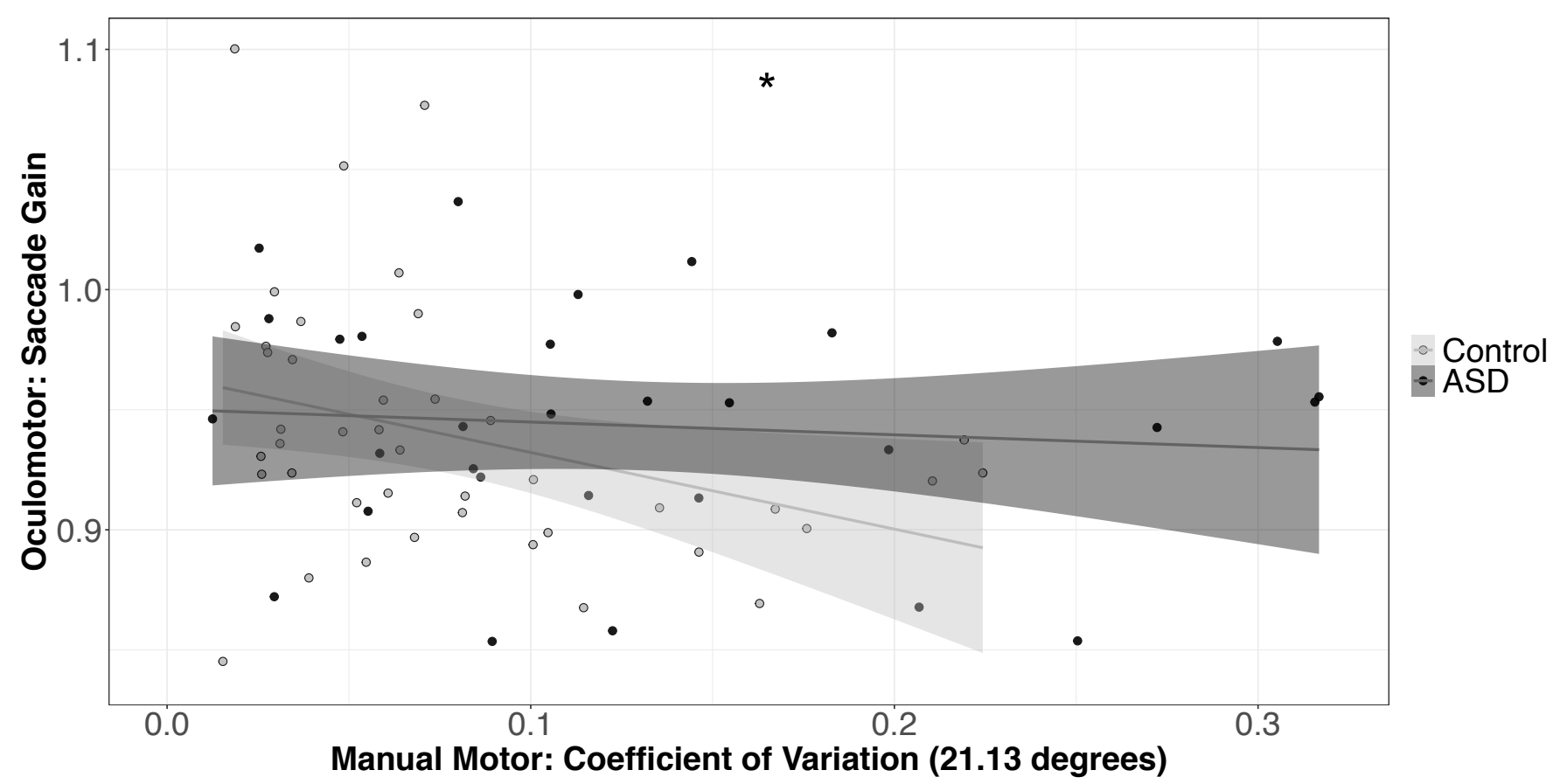

Figure 8. Increased saccade accuracy was associated with reduced $\mathrm{CoV}$ in controls, but not in individuals with ASD, across multiple task conditions (Gain task: 21.13 degrees depicted). Error bars reflect standard error of the mean. *slopes differ between groups.

\section{Clinical Correlations}

Among individuals with ASD, more severe communication abnormalities (ADI-R) were associated with increased CoV at high force $(r=.30, p=.03)$ and increased saccade dysmetria $(r=.30, p=.01)$. More severe clinically-rated RRBs also were associated with increased force error (RBS-R; $r=.39, p=.01)$, increased CoV at high force (RBS-R; $r=.26, p=$ $.04)$, and increased saccade dysmetria (ADI-R; $r=.33, p=.01$ ). 


\section{Discussion}

This is the first known study to quantify sensorimotor deficits in individuals with ASD across multiple effectors (hand and eye) and motor control processes, including feedforward and feedback mechanisms. Four key findings emerged. First, we demonstrate that individuals with ASD show increases in manual motor variability and motor regularity (ApEn) relative to TD that are strongly age-dependent. Interestingly, alterations in motor variability and regularity in ASD show distinct age-associations. Specifically, early increases in fine motor control variability in ASD showed marked improvements, while increased regularity was more pronounced in older individuals with ASD relative to age-matched controls, suggesting that separate neurodevelopmental mechanisms may contribute to these different motor deficits. Second, saccade accuracy is reduced in ASD, especially during adolescence and adulthood suggesting dysfunctions of feedforward control processes become more severe over development. Third, grip and saccade behaviors are related in TD controls, but not in ASD, suggesting independence of feedforward and feedback motor deficits in ASD. Fourth, increased force variability and saccade dysmetria were associated with the severity of communication and RRB symptoms of ASD suggesting the neurodevelopmental processes associated with both feedback and feedforward motor behaviors may play a central role in the development of core ASD features.

\section{Continuous control of motor behavior in ASD}

Consistent with prior studies, we found that individuals with ASD show increased sensorimotor variability relative to controls suggesting reduced ability to translate sensory feedback to guide ongoing motor behavior (Mosconi et al., 2015; Unruh et al., 2019; Wang et al., 2015). We also show that failure to precisely adjust motor behavior during precision gripping at lower force levels is more severely disrupted in younger individuals with ASD. For example, we found minimal overlap in force variability at 15\% MVC between individuals with ASD and controls ages 5-10 years. The majority of daily activities involve precision manual manipulations involving force output between 5-15\% MVC suggesting that early developmental difficulties could significantly disrupt multiple developmental behaviors. These findings are consistent with prior studies suggesting sensorimotor issues emerge early in ASD and are more prominent during early childhood relative to adolescence and adulthood (Freitag et al., 2007; Luna et al., 2007). Early emerging issues in sensory feedback control of motor behavior could reflect atypical sensory and multisensory processing as suggested by prospective studies demonstrating rapid over-expansion of sensory cortex in infants who are later diagnosed with ASD (Hazlett et al., 2017). Importantly, these results also suggest that measurement of precision gripping variability 
at low force levels may help reliably differentiate children with ASD and age-matched TD children, in line with recent data demonstrating that early emerging impairments in fine motor, but not gross motor, abilities were predictive of later ASD severity (Iverson et al., 2019). Deficits in early motor development in ASD appear to be heritable and predictive of familial recurrence suggesting that tracking select motor behaviors may provide important insights into both early emerging neurodevelopmental mechanisms and important targets for monitoring early emerging ASD risk factors (Hilton et al., 2012; Libertus et al., 2014; Pohl et al., 2019).

Dynamic adaptation of behavior is the product of integrated inputs across multiple modalities that operate on varying timescales, including rapid feedforward processes and slower visual, proprioceptive, and haptic feedback information. The integration of these multiple control processes is reflected in the time-dependent structure of the motor output, with greater irregularity reflecting greater integration of independent systems (e.g., Pincus \& Goldberger, 1994). Consistent with our previous studies, we found reduced ApEn in ASD relative to controls suggesting a diminished ability to integrate feedforward and sensory feedback processes that operate on different time scales (Mosconi, Mohanty, et al., 2015). A tight coupling between force variability and irregularity indicates a strong contribution of sensory feedback to precision motor output (Slifkin \& Newell, 1999), as demonstrated by our data showing a strong inverse correlation between CoV and ApEn across participants at low and medium force levels. At high force, individuals with ASD demonstrated reduced coupling of force variability and irregularity relative to controls, implicating reduced capacity to integrate multiple feedback and feedforward processes to mitigate variability under more challenging conditions. Similarly, deficits in ApEn were more pronounced in ASD at high levels of force and when using the nondominant hand. Together these results suggest that as demand on the motor system increases, individuals with ASD may become less reliant on integrated sensory inputs and increasingly reliant on discrete primary or dominant mechanisms such as internal action plans or visual sensory feedback for precision motor control (Haswell et al., 2009; Mosconi, Mohanty, et al., 2015). Our findings of distinct age-associations in motor variability and regularity in individuals with ASD suggest a dissociation of motor control processes that may reflect multiple neurodevelopmental alterations contributing to impairment of continuous motor behaviors in ASD. Finally, we found that females with ASD showed relatively preserved sensorimotor abilities at low force suggesting sex-specific impacts on motor development. Though it is important to note that our study included only a small number of females with ASD, these data are consistent with findings of sex-specific morphological differences in sensory and motor brain regions in ASD (Supekar \& Menon, 2015) and opposing patterns of hypo- 
connectivity in males compared to hyper-connectivity in females with ASD within cerebellar sensory-motor networks (Smith et al., 2019). While ASD disproportionately affects males, our findings highlight the need for future research on sensorimotor development in ASD to include females.

\section{Rapid motor control in ASD}

We demonstrate reduced saccade accuracy in individuals with ASD, implicating deficits in internal models that support precise motor control. Internal models are formed as a result of repeated engagement in a behavior and refined via corrective error signals (Ito, 2008). In the case of eye movements, sensory feedback is used to modulate the amplitude or duration of the subsequent saccade to minimize endpoint error (Ohtsuka \& Noda, 1992; Pelisson \& Prablanc, 1988). Increased saccade dysmetria suggests reduced precision of the internal model in ASD that may arise from deficiencies in consolidation of sensory feedback, consistent with previous reports of increased trial-to-trial saccade error (Luna et al., 2007; Schmitt et al., 2014; Takarae et al., 2004) and reduced adaptation of saccade amplitudes following experimentally induced error (Mosconi et al., 2013). Reduced age-associated improvements in saccade accuracy in ASD appear to reflect attenuation of maturational processes that support refinement of feedforward motor control processes into early adulthood as was seen in healthy controls. Our results contrast with those of previous studies that have demonstrated reduced saccade accuracy that is more severe in children, compared to adults (Luna et al., 2007; Takarae et al., 2004). As we also find that saccade dysmetria covaries with the severity of core ASD symptoms, it is possible that differences between our results and those of Luna et al. may reflect distinctions in the severity of individuals studied. This possibility is further emphasized by findings that saccade accuracy varies as a function of language delay in ASD (Takarae et al., 2004). Longitudinal studies of saccade accuracy across a range of severity of core and associated symptoms will help clarify patterns of maturation of feedforward motor control processes.

In contrast to prior studies reporting that latencies are relatively unaffected in ASD (Luna et al., 2007; Minshew et al., 1999; Schmitt et al., 2014), in our study with a larger cohort, we found that individuals with ASD showed decreased saccade latency relative to controls. Effect sizes for these group comparisons were small $(d \mathrm{~s}=.11-.19)$ relative to the variation across individuals in this metric (see Supplementary Figure 3). Studies modulating attentional capture are needed to clarify mechanisms of atypical saccade reaction times in ASD. 


\section{Relationships between deficits in feedback and feedforward processes in ASD}

We failed to identify significant relationships between deficits in feedback and feedforward motor control processes in ASD. Along with our data showing feedback deficits in ASD were more severe during early childhood while impairments in feedforward processes were more severe at older ages, these findings suggest that separate sensorimotor deficits involve distinct neurodevelopmental mechanisms. Feedforward and feedback-guided processes are governed by separate, but integrated brain systems that are thought to operate along a continuum (Seidler et al., 2004). Rapid, feedforward behaviors primarily are controlled by highly precise internal models which are thought to be refined and stored in the cerebellum and initiated via prefrontal cortex (Ito, 2008). In contrast, continuous motor behaviors are controlled by reciprocal network connections involving sensory cortical innervation of cerebellar circuits that mediate dynamic adjustment of ongoing motor commands generated in primary motor cortex (Desmurget \& Grafton, 2000). Using visualspatial information from occipital and parietal cortices, the cerebellum computes the difference between predicted sensory consequences of an action and real-time sensory feedback and relays this motor error information to motor cortex to refine continuous behavior (Stein \& Glickstein, 1992). Consistent with this model, prior fMRI studies have shown increased motor variability in ASD is associated with increased activation of posterior cerebellum, reduced activations of posterior parietal and premotor cortices (Unruh et al., 2019), and increased cerebellar functional connectivity with visual cortex (Wang et al., 2019). Our findings implicate dysfunction of multiple cortical-cerebellar networks and suggest that these separate networks may be independently affected. This inference is supported both by their low correlation and by their differential developmental trajectory. Results showing that deficits in feedback motor control processes become less severe during childhood implicate delayed maturation of visual cortical-cerebellar circuits, or reorganization of networks to accommodate for early emerging sensory-motor disturbances as is observed in other motor disorders (e.g., Parkinson's disease and cerebellar degeneration; Sabatini et al., 2000; Wessel et al., 1995). Consistent with the hypothesis that separate "non-motor" networks may compensate for early developmental dysmaturation of cortical-cerebellar systems, Takarae et al (2007) demonstrated increased activation of frontal-striatal circuits typically involved in higher-order cognition and the voluntary control of behavior during visually-guided saccades in ASD. Longitudinal studies are needed to characterize the maturation of cortical-cerebellar systems in ASD to determine whether distinct sensorimotor issues arise from developmental delays that are attenuated over time, or represent deviations from typical neurodevelopment that may be compensated for by other systems. 


\section{Limitations and implications for future research}

A primary limitation of this study is the reliance on cross-sectional rather than longitudinal data. While our large sample spanning a wide age-range provides important landmarks for tracking change in feedforward and feedback motor control systems, longitudinal studies of separate motor skills and their association with functional outcomes are needed to clarify neurodevelopmental mechanisms of core and associated symptoms in ASD. A second limitation of our study is that the majority of our participants with ASD had average to above average IQs; therefore, these results may not be generalizable to individuals with ASD and comorbid intellectual/developmental disability. While we did not see strong associations between cognitive ability and motor behavior in our sample, it is possible that sensorimotor issues may covary more strongly when studying individuals outside of the normal range of IQ as suggested previously (Travers et al., 2013, 2017). Finally, our examination of feedforward mechanisms focused on eye movements whereas our analysis of feedback processes assessed manual behavior. Ballistic eye movements offer a unique and critical target for quantifying feedforward motor control as they are completed too rapidly to be guided by online feedback adjustments. In contrast, manual behaviors progress over a relatively protracted time scale but are regularly modified in response to sensory feedback. Still, this design limits our ability to determine whether patterns of age-associated differences in feedforward and feedback processes each vary across effectors.

\section{Conclusions}

This study is among the first to characterize both rapid and continuous motor behaviors and age-associated changes in individuals with ASD across a wide range of development. Our data indicate feedforward and feedback processes are disrupted in ASD and follow separate neurodevelopmental patterns. These results highlight the strong need to parse separate sensorimotor behaviors and track them longitudinally to define spared and affected systems in ASD and track their change during development. Together with prior studies showing that motor deficits are common and early emerging in ASD, our findings of impairments in precision motor behavior in young children with ASD that covary with core communication symptoms highlight the potential utility of tracking select sensorimotor behaviors to promote earlier and more objective identification strategies. 


\section{List of abbreviations}

ASD, Autism Spectrum Disorder

TD, typically developing

UIC, University of Illinois at Chicago

UTSW, University of Texas Southwestern Medical Center

DAS, Differential Ability Scale

WASI, Wechsler Abbreviated Scale of Intelligence

WPPSI, Wechsler Preschool and Primary Scales of Intelligence

ADI-R, Autism Diagnostic Inventory-Revised

ADOS, Autism Diagnostic Observation Schedule - Second Edition

DSM, Diagnostic and Statistical Manual of Mental Disorders

MVC, maximum voluntary contraction

$\mathrm{CoV}$, coefficient of variation

ApEn, approximate entropy

$\mathrm{SD}$, standard deviation

CSS, calibrated severity score

RBS-R, Repetitive Behavior Scale-Revised

VGS, visually-guided saccade

\section{Declarations}

Ethics approval and consent to participate: These studies were approved by the Institutional Review Boards at the University of Illinois at Chicago and University of Texas Southwestern Medical Center. Written informed consent was obtained from all participants, with assent and parental consent obtained for minors.

Consent for publication: Not applicable

Availability of data and materials: The datasets used and/or analyzed during the current study are available from the corresponding author on reasonable request. 
Competing interests: Dr. Sweeney consults to VeraSci.

Funding: This study was funded by grants NICHD U54 090216, NIMH R01 112734, and Kansas Center for Autism Research and Training (K-CART) Research Investment Council Strategic Initiative Grant.

Author contributions: MWM and JAS conceived and designed the research. MWM performed the experiments. KEU, WSM, and KKF designed and performed the data analysis. KEU, MWM, WSM, and KKF interpreted the results. KEU drafted the manuscript. JAS, MWM, WSM, and KKF edited and revised the manuscript. All authors read and approved the final manuscript. 
Allen, G., \& Courchesne, E. (2003). Differential Effects of Developmental Cerebellar Abnormality on Cognitive and Motor Functions in the Cerebellum: An fMRI Study of Autism. American Journal of Psychiatry, 160(2), 262273. https://doi.org/10.1176/appi.ajp.160.2.262

Allen, G., Müller, R.-A., \& Courchesne, E. (2004). Cerebellar function in autism: Functional magnetic resonance image activation during a simple motor task. Biological Psychiatry, 56(4), 269-278.

Bates, D., Mächler, M., Bolker, B., \& Walker, S. (2015). Fitting Linear Mixed-Effects Models Using Ime4. Journal of Statistical Software, 67(1). https://doi.org/10.18637/jss.v067.i01

Bojanek, E. K., Wang, Z., White, S. P., \& Mosconi, M. W. (2020). Postural control processes during standing and step initiation in autism spectrum disorder. Journal of Neurodevelopmental Disorders, 12(1), 1. https://doi.org/10.1186/s11689-019-9305-x

Bryson, S. E., Zwaigenbaum, L., Brian, J., Roberts, W., Szatmari, P., Rombough, V., \& McDermott, C. (2007). A prospective case series of high-risk infants who developed autism. Journal of Autism and Developmental Disorders, 37(1), 12-24. http://link.springer.com.proxy.library.vanderbilt.edu/article/10.1007/s10803-006-0328-2 Desmurget, M., \& Grafton, S. (2000). Forward modeling allows feedback control for fast reaching movements. Trends in Cognitive Sciences, 4(11), 423-431. http://www.sciencedirect.com.proxy.library.vanderbilt.edu/science/article/pii/S1364661300015370

Dowell, L. R., Mahone, E. M., \& Mostofsky, S. H. (2009). Associations of postural knowledge and basic motor skill with dyspraxia in autism: Implication for abnormalities in distributed connectivity and motor learning. Neuropsychology, 23(5), 563-570. https://doi.org/10.1037/a0015640

Dziuk, M. A., Larson, J. C., Apostu, A., Mahone, E. M., Denckla, M. B., \& Mostofsky, S. H. (2007). Dyspraxia in autism: Association with motor, social, and communicative deficits. Developmental Medicine \& Child Neurology, 49(10), 734-739. http://onlinelibrary.wiley.com.proxy.library.vanderbilt.edu/doi/10.1111/j.1469-8749.2007.00734.x/full

Elberling, H., Linneberg, A., Olsen, E. M., Houmann, T., Rask, C. U., Goodman, R., \& Skovgaard, A. M. (2014). Infancy predictors of hyperkinetic and pervasive developmental disorders at ages 5-7 years: Results from the Copenhagen Child Cohort CCC2000. Journal of Child Psychology and Psychiatry, 55(12), 1328-1335. http://onlinelibrary.wiley.com.proxy.library.vanderbilt.edu/doi/10.1111/jcpp.12256/full

Fatemi, S. H., Aldinger, K. A., Ashwood, P., Bauman, M. L., Blaha, C. D., Blatt, G. J., Chauhan, A., Chauhan, V., Dager, S. R., Dickson, P. E., Estes, A. M., Goldowitz, D., Heck, D. H., Kemper, T. L., King, B. H., Martin, L. A., Millen, 
K. J., Mittleman, G., Mosconi, M. W., ... Welsh, J. P. (2012). Consensus Paper: Pathological Role of the Cerebellum in Autism. The Cerebellum, 11(3), 777-807. https://doi.org/10.1007/s12311-012-0355-9

Fournier, K. A., Kimberg, C. I., Radonovich, K. J., Tillman, M. D., Chow, J. W., Lewis, M. H., Bodfish, J. W., \& Hass, C. J. (2010). Decreased static and dynamic postural control in children with autism spectrum disorders. Gait \& Posture, 32(1), 6-9. http://www.sciencedirect.com.proxy.library.vanderbilt.edu/science/article/pii/S0966636210000603

Freitag, C. M., Kleser, C., Schneider, M., \& von Gontard, A. (2007). Quantitative assessment of neuromotor function in adolescents with high functioning autism and Asperger syndrome. Journal of Autism and Developmental Disorders, 37(5), 948-959.

Glazebrook, C., Elliott, D., \& Lyons, J. (2006). A kinematic analysis of how young adults with and without autism plan and control goal-directed movements. Motor Control, 10(3), 244-264.

Glazebrook, C., Gonzalez, D., Hansen, S., \& Elliott, D. (2009). The role of vision for online control of manual aiming movements in persons with autism spectrum disorders. Autism, 13(4), 411-433.

Gotham, K., Pickles, A., \& Lord, C. (2009). Standardizing ADOS Scores for a Measure of Severity in Autism Spectrum Disorders. Journal of Autism and Developmental Disorders, 39(5), 693-705. https://doi.org/10.1007/s10803-0080674-3

Haswell, C. C., Izawa, J., Dowell, L. R., Mostofsky, S. H., \& Shadmehr, R. (2009). Representation of internal models of action in the autistic brain. Nature Neuroscience, 12(8), 970-972. https://doi.org/10.1038/nn.2356

Hazlett, H. C., Gu, H., Munsell, B. C., Kim, S. H., Styner, M., Wolff, J. J., Elison, J. T., Swanson, M. R., Zhu, H., \& Botteron, K. N. (2017). Early brain development in infants at high risk for autism spectrum disorder. Nature, 542(7641), 348.

Hilton, C. L., Zhang, Y., Whilte, M. R., Klohr, C. L., \& Constantino, J. (2012). Motor impairment in sibling pairs concordant and discordant for autism spectrum disorders. Autism, 16(4), 430-441. https://doi.org/10.1177/1362361311423018

Hox, J. J., Moerbeek, M., \& Schoot, R. van de. (2017). Multilevel Analysis: Techniques and Applications, Third Edition. Routledge.

Ito, M. (2008). Control of mental activities by internal models in the cerebellum. Nature Reviews Neuroscience, 9(4), 304-313. https://doi.org/10.1038/nrn2332 
Iverson, J. M., Shic, F., Wall, C. A., Chawarska, K., Curtin, S., Estes, A., Gardner, J. M., Hutman, T., Landa, R. J., Levin, A. R., Libertus, K., Messinger, D. S., Nelson, C. A., Ozonoff, S., Sacrey, L.-A. R., Sheperd, K., Stone, W. L., Tager-Flusberg, H. B., Wolff, J. J., ... Young, G. S. (2019). Early motor abilities in infants at heightened versus low risk for ASD: A Baby Siblings Research Consortium (BSRC) study. Journal of Abnormal Psychology, 128(1), 69-80. https://doi.org/10.1037/abn0000390

Koller, M. (2016). robustlmm: An R Package for Robust Estimation of Linear Mixed-Effects Models. Journal of Statistical Software, 75(6), 1-24. https://doi.org/10.18637/jss.v075.i06

Kuznetsova, A., Brockhoff, P. B., \& Christensen, R. H. B. (2017). lmerTest Package: Tests in Linear Mixed Effects Models. Journal of Statistical Software, 82(13), 1-26. https://doi.org/10.18637/jss.v082.i13

Lam, K. S., \& Aman, M. G. (2007). The Repetitive Behavior Scale-Revised: Independent validation in individuals with autism spectrum disorders. Journal of Autism and Developmental Disorders, 37(5), 855-866.

Libertus, K., Sheperd, K. A., Ross, S. W., \& Landa, R. J. (2014). Limited Fine Motor and Grasping Skills in 6-Month-Old Infants at High Risk for Autism. Child Development, 85(6), 2218-2231. https://doi.org/10.1111/cdev.12262

Lord, C., Risi, S., Lambrecht, L., Cook Jr, E. H., Leventhal, B. L., DiLavore, P. C., Pickles, A., \& Rutter, M. (2000). The Autism Diagnostic Observation Schedule - Generic: A standard measure of social and communication deficits associated with the spectrum of autism. Journal of Autism and Developmental Disorders, 30(3), 205-223.

Lord, C., Rutter, M., \& Le Couteur, A. (1994). Autism Diagnostic Interview-Revised: A revised version of a diagnostic interview for caregivers of individuals with possible pervasive developmental disorders. Journal of Autism and Developmental Disorders, 24(5), 659-685.

Luna, B., Doll, S. K., Hegedus, S. J., Minshew, N. J., \& Sweeney, J. A. (2007). Maturation of Executive Function in Autism. Biological Psychiatry, 61(4), 474-481. https://doi.org/10.1016/j.biopsych.2006.02.030

Luna, B., Garver, K. E., Urban, T. A., Lazar, N. A., \& Sweeney, J. A. (2004). Maturation of Cognitive Processes From Late Childhood to Adulthood. Child Development, 75(5), 1357-1372. https://doi.org/10.1111/j.14678624.2004.00745.x

Manicolo, O., Brotzmann, M., Hagmann-von Arx, P., Grob, A., \& Weber, P. (2019). Gait in children with infantile/atypical autism: Age-dependent decrease in gait variability and associations with motor skills. European Journal of Paediatric Neurology, 23(1), 117-125. https://doi.org/10.1016/j.ejpn.2018.09.011 
Matuschek, H., Kliegl, R., Vasishth, S., Baayen, H., \& Bates, D. (2017). Balancing Type I error and power in linear mixed models. Journal of Memory and Language, 94, 305-315. https://doi.org/10.1016/j.jml.2017.01.001

Minshew, N. J., Luna, B., \& Sweeney, J. A. (1999). Oculomotor evidence for neocortical systems but not cerebellar dysfunction in autism. Neurology, 52(5), 917-922. https:/www.ncbi.nlm.nih.gov/pmc/articles/PMC2995853/

Mosconi, M. W., Kay, M., D’Cruz, A.-M., Guter, S., Kapur, K., Macmillan, C., Stanford, L. D., \& Sweeney, J. A. (2010). Neurobehavioral abnormalities in first-degree relatives of individuals with autism. Archives of General Psychiatry, 67(8), 830-840. http://jamanetwork.com.proxy.library.vanderbilt.edu/journals/jamapsychiatry/fullarticle/210853

Mosconi, M. W., Luna, B., Kay-Stacey, M., Nowinski, C. V., Rubin, L. H., Scudder, C., Minshew, N., \& Sweeney, J. A. (2013). Saccade Adaptation Abnormalities Implicate Dysfunction of Cerebellar-Dependent Learning Mechanisms in Autism Spectrum Disorders (ASD). PLOS ONE, 8(5), e63709. https://doi.org/10.1371/journal.pone.0063709

Mosconi, M. W., Mohanty, S., Greene, R. K., Cook, E. H., Vaillancourt, D. E., \& Sweeney, J. A. (2015). Feedforward and feedback motor control abnormalities implicate cerebellar dysfunctions in autism spectrum disorder. Journal of Neuroscience, 35(5). http://www.jneurosci.org.proxy.library.vanderbilt.edu/content/35/5/2015.short

Mosconi, M. W., Wang, Z., Schmitt, L. M., Tsai, P., \& Sweeney, J. A. (2015). The role of cerebellar circuitry alterations in the pathophysiology of autism spectrum disorders. Frontiers in Neuroscience, 9. https://doi.org/10.3389/fnins.2015.00296

Ohtsuka, K., \& Noda, H. (1992). Burst discharges of mossy fibers in the oculomotor vermis of macaque monkeys during saccadic eye movements. Neuroscience Research, 15(1), 102-114. https://doi.org/10.1016/0168-0102(92)90023-6

Pelisson, D., \& Prablanc, C. (1988). Kinematics of centrifugal and centripetal saccadic eye movements in man. Vision Research, 28(1), 87-94. https://doi.org/10.1016/S0042-6989(88)80009-9

Pincus, S. M., \& Goldberger, A. L. (1994). Physiological time-series analysis: What does regularity quantify? American Journal of Physiology-Heart and Circulatory Physiology, 266(4), H1643-H1656. https://doi.org/10.1152/ajpheart.1994.266.4.H1643

Pohl, A., Jones, W. R., Marrus, N., Zhang, Y., Klin, A., \& Constantino, J. N. (2019). Behavioral predictors of autism recurrence are genetically independent and influence social reciprocity: Evidence that polygenic ASD risk is mediated by separable elements of developmental liability. Translational Psychiatry, 9(1), 1-9. https://doi.org/10.1038/s41398-019-0545-z 
Reilly, J. L., Lencer, R., Bishop, J. R., Keedy, S., \& Sweeney, J. A. (2008). Pharmacological treatment effects on eye movement control. Brain and Cognition, 68(3), 415-435.

Robichaud, J. A., Pfann, K. D., Vaillancourt, D. E., Comella, C. L., \& Corcos, D. M. (2005). Force control and disease severity in Parkinson's disease. Movement Disorders, 20(4), 441-450.

Sabatini, U., Boulanouar, K., Fabre, N., Martin, F., Carel, C., Colonnese, C., Bozzao, L., Berry, I., Montastruc, J. L., Chollet, F., \& Rascol, O. (2000). Cortical motor reorganization in akinetic patients with Parkinson's disease: A functional MRI study. Brain, 123(2), 394-403. https://doi.org/10.1093/brain/123.2.394

Schmitt, L. M., Cook, E. H., Sweeney, J. A., \& Mosconi, M. W. (2014). Saccadic eye movement abnormalities in autism spectrum disorder indicate dysfunctions in cerebellum and brainstem. Molecular Autism, 5(1), 47.

Seidler, R., Noll, D., \& Thiers, G. (2004). Feedforward and feedback processes in motor control. Neuroimage, 22(4), $1775-1783$.

Slifkin, A. B., \& Newell, K. M. (1999). Noise, information transmission, and force variability. Journal of Experimental Psychology: Human Perception and Performance, 25(3), 837-851. https://doi.org/10.1037/0096-1523.25.3.837

Smith, R. E. W., Avery, J. A., Wallace, G. L., Kenworthy, L., Gotts, S. J., \& Martin, A. (2019). Sex Differences in Resting-State Functional Connectivity of the Cerebellum in Autism Spectrum Disorder. Frontiers in Human Neuroscience, 13. https://doi.org/10.3389/fnhum.2019.00104

Stein, J. F., \& Glickstein, M. (1992). Role of the cerebellum in visual guidance of movement. Physiological Reviews, 72(4), 967-1017. https://doi.org/10.1152/physrev.1992.72.4.967

Supekar, K., \& Menon, V. (2015). Sex differences in structural organization of motor systems and their dissociable links with repetitive/restricted behaviors in children with autism. Molecular Autism, 6(1). https://doi.org/10.1186/s13229-015-0042-z

Sweeney, J. A., Takarae, Y., Macmillan, C., Luna, B., \& Minshew, N. J. (2004). Eye movements in neurodevelopmental disorders. Current Opinion in Neurology, 17(1), 37-42. https://journals.lww.com/coneurology/Abstract/2004/02000/Eye_movements_in_neurodevelopmental_disorders.7.aspx

Takarae, Y., Minshew, N. J., Luna, B., \& Sweeney, J. A. (2004). Oculomotor abnormalities parallel cerebellar histopathology in autism. Journal of Neurology, Neurosurgery \& Psychiatry, 75(9), 1359-1361. https://doi.org/10.1136/jnnp.2003.022491 
Takarae, Yukari, Minshew, N. J., Luna, B., \& Sweeney, J. A. (2007). Atypical involvement of frontostriatal systems during sensorimotor control in autism. Psychiatry Research: Neuroimaging, 156(2), 117-127.

Travers, B. G., Bigler, E. D., Duffield, T. C., Prigge, M. D., Froehlich, A. L., Lange, N., Alexander, A. L., \& Lainhart, J. E. (2017). Longitudinal development of manual motor ability in autism spectrum disorder from childhood to midadulthood relates to adaptive daily living skills. Developmental Science, 20(4). http://onlinelibrary.wiley.com.proxy.library.vanderbilt.edu/doi/10.1111/desc.12401/full

Travers, B. G., Powell, P. S., Klinger, L. G., \& Klinger, M. R. (2013). Motor difficulties in autism spectrum disorder: Linking symptom severity and postural stability. Journal of Autism and Developmental Disorders, 43(7), 15681583. http://link.springer.com.proxy.library.vanderbilt.edu/article/10.1007/s10803-012-1702-x

Unruh, K. E., Martin, L. E., Magnon, G., Vaillancourt, D. E., Sweeney, J. A., \& Mosconi, M. W. (2019). Cortical and subcortical alterations associated with precision visuomotor behavior in individuals with autism spectrum disorder. Journal of Neurophysiology, 122(4), 1330-1341. https://doi.org/10.1152/jn.00286.2019

Vaillancourt, D. E., Slifkin, A. B., \& Newell, K. M. (2001). Regularity of force tremor in Parkinson's disease. Clinical Neurophysiology, 112(9), 1594-1603. https://doi.org/10.1016/S1388-2457(01)00593-4

Vaillancourt, D. E., Thulborn, K. R., \& Corcos, D. M. (2003). Neural basis for the processes that underlie visually guided and internally guided force control in humans. Journal of Neurophysiology, 90(5), 3330-3340. http://jn.physiology.org.proxy.library.vanderbilt.edu/content/90/5/3330.short

Wang, Z., Hallac, R. R., Conroy, K. C., White, S. P., Kane, A. A., Collinsworth, A. L., Sweeney, J. A., \& Mosconi, M. W. (2016). Postural orientation and equilibrium processes associated with increased postural sway in autism spectrum disorder (ASD). Journal of Neurodevelopmental Disorders, 8(1), 43. https://jneurodevdisorders-biomedcentralcom.proxy.library.vanderbilt.edu/articles/10.1186/s11689-016-9178-1

Wang, Z., Magnon, G. C., White, S. P., Greene, R. K., Vaillancourt, D. E., \& Mosconi, M. W. (2015). Individuals with autism spectrum disorder show abnormalities during initial and subsequent phases of precision gripping. Journal of Neurophysiology, 113(7), 1989-2001. http://jn.physiology.org.proxy.library.vanderbilt.edu/content/113/7/1989.abstract

Wang, Z., Wang, Y., Sweeney, J. A., Gong, Q., Lui, S., \& Mosconi, M. W. (2019). Resting-State Brain Network Dysfunctions Associated With Visuomotor Impairments in Autism Spectrum Disorder. Frontiers in Integrative Neuroscience, 13. https://doi.org/10.3389/fnint.2019.00017 
Wessel, K., Zeffiro, T., Lou, J.-S., Toro, C., \& Hallett, M. (1995). Regional cerebral blood flow during a self-paced sequential finger opposition task in patients with cerebellar degeneration. Brain, 118(2), 379-393.

https://doi.org/10.1093/brain/118.2.379 


\section{Figures}

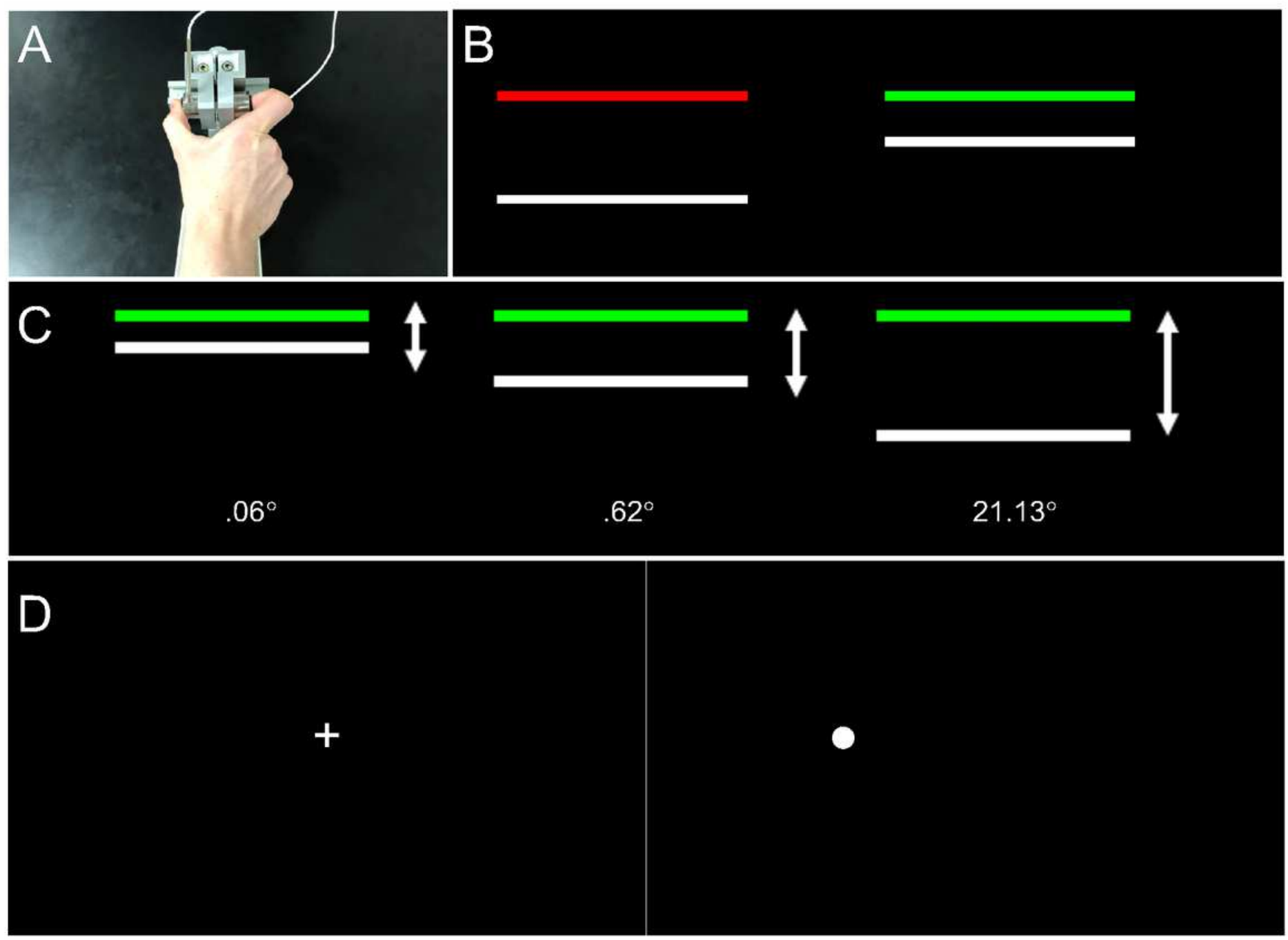

Figure 1

A. Precision grip load cell apparatus. B. Precision grip stimuli. Participants viewed a red TARGET bar and white FORCE bar. Once the TARGET bar turned green, participants pressed the load cells to bring the white FORCE bar up to the level of the green TARGET bar. C. Precision grip visual gain manipulation. The amount of visual feedback was either degraded (left: $0.06^{\circ}$ ) or amplified (rightmost: $21.13^{\circ}$ ). D. Visually guided saccade stimuli. Participants viewed crosshairs and made a saccade to the left $\left(-12^{\circ}\right.$; depicted) or right $\left(+12^{\circ}\right)$. 


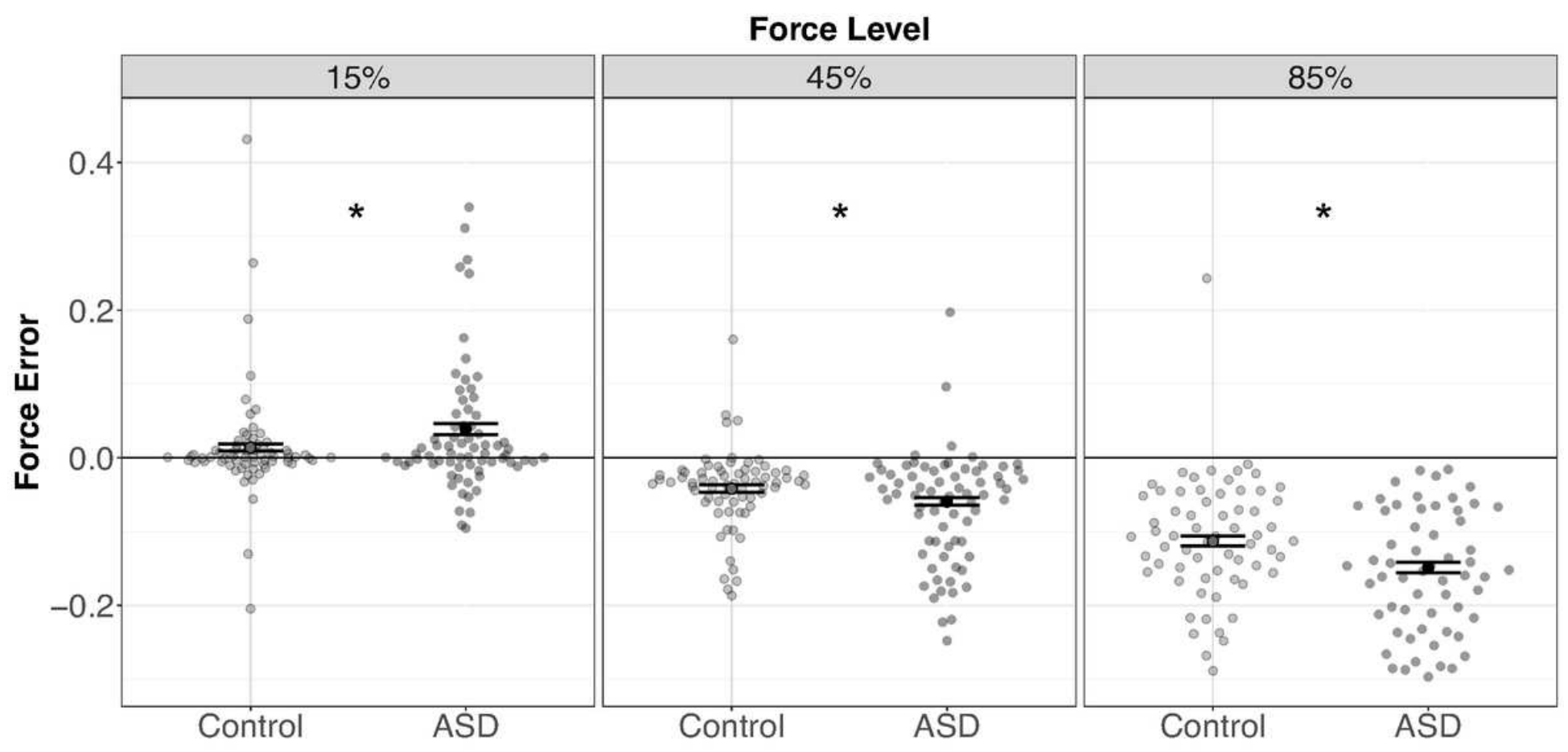

Figure 2

Force error was increased in ASD participants relative to TD controls across all levels of force. Compared to controls, individuals with ASD demonstrated target overshoot at $15 \%$ and target undershoot at 45 and $85 \%$ MVC. Error bars reflect standard error of the mean. ${ }^{*} p<.05$. 


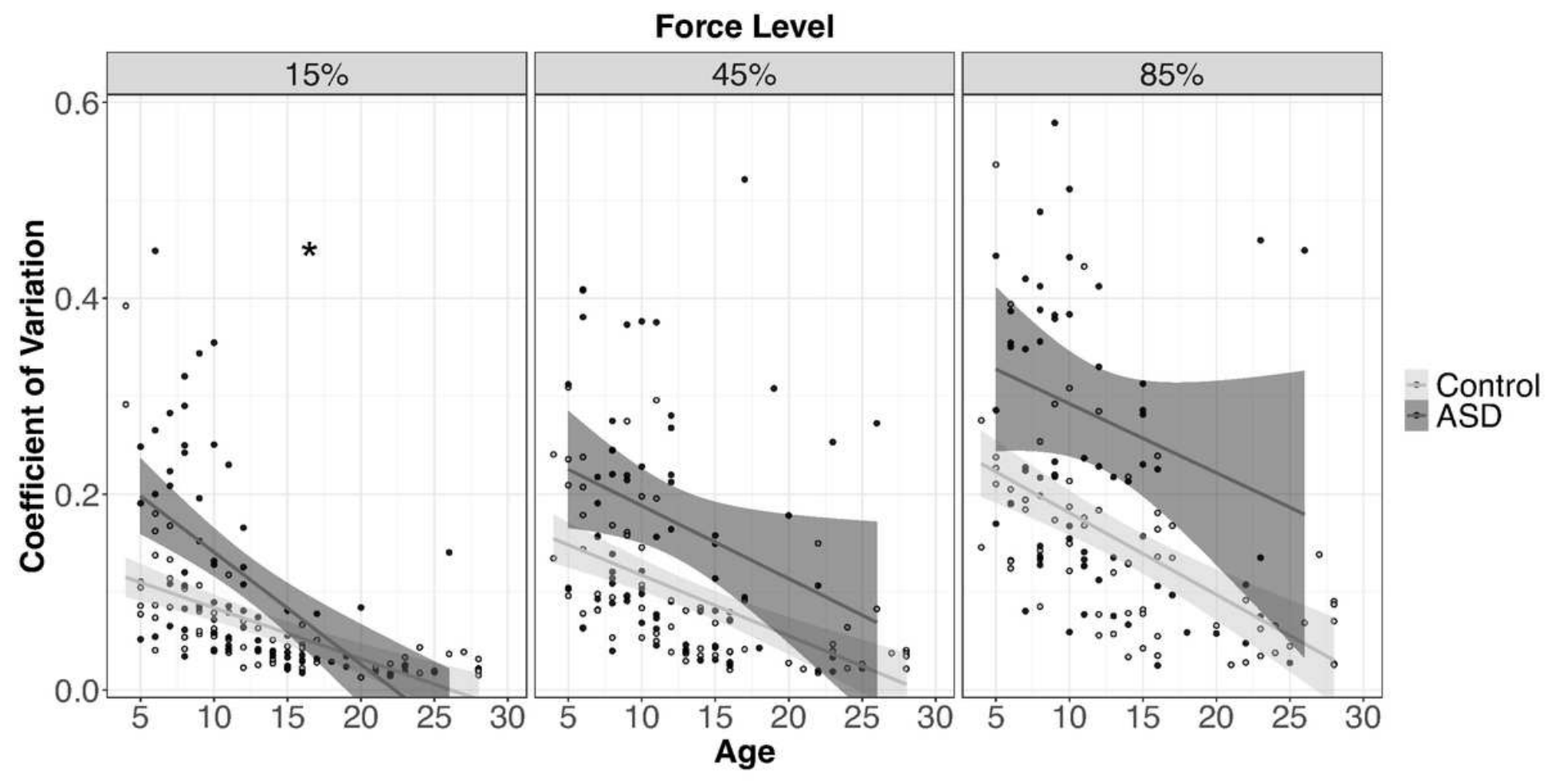

Figure 3

Force variability was increased in ASD participants relative to TD controls. Elevations in force variability were more severe in younger individuals with ASD relative to older individuals with ASD at $15 \%$ MVC. Error bars reflect standard error of the mean. *slopes differ between groups. ${ }^{*} p<.05$. 


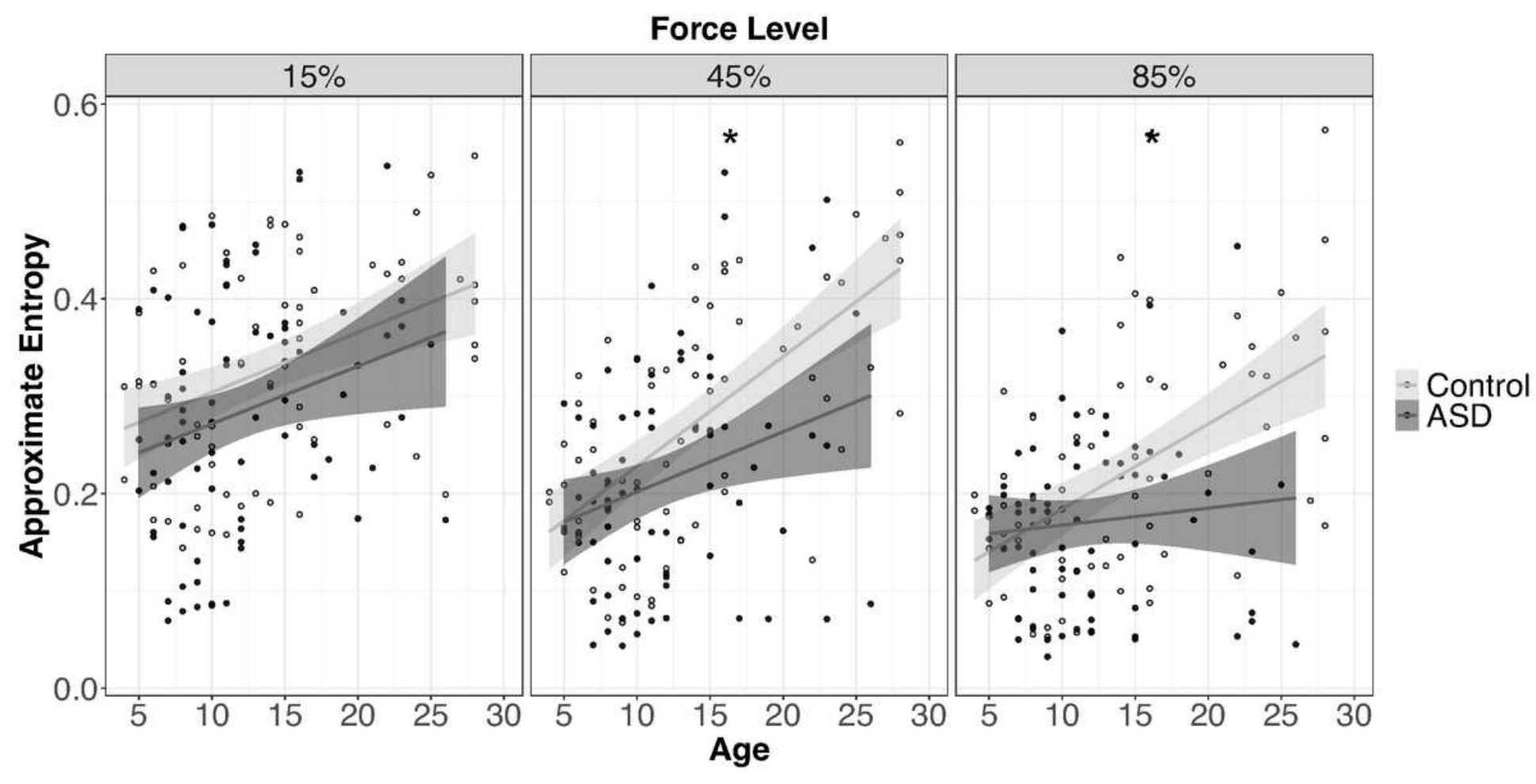

Figure 4

Approximate entropy is reduced in individuals with ASD compared to controls across all force levels. At 45 and $85 \%$ MVC, individuals with ASD show reduced age-associated increases in ApEn compared to controls. Error bars reflect standard error of the mean. *slopes differ between groups. 


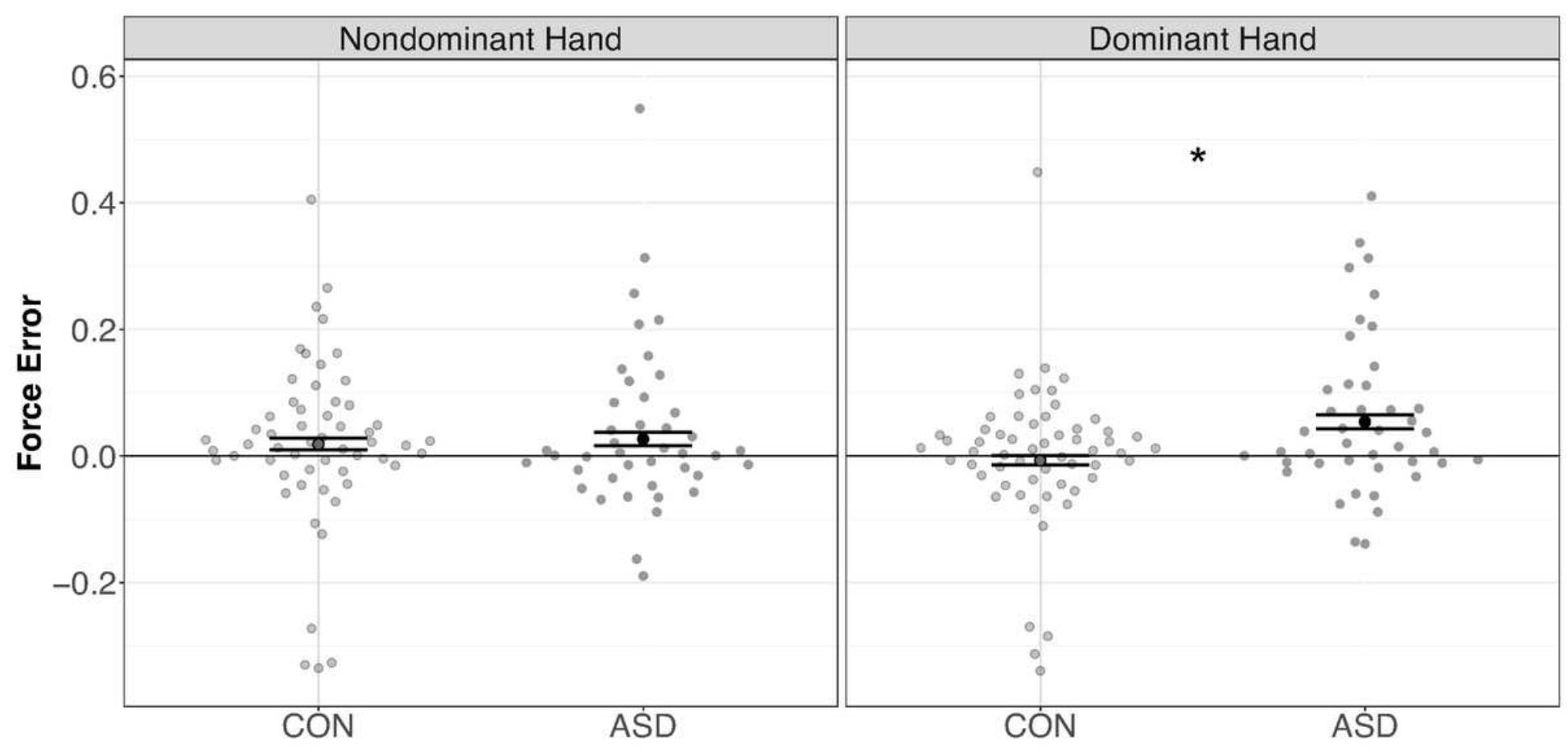

Figure 5

Force error was increased in ASD participants relative to TD controls when using the dominant hand. Negative values indicate target undershoot and positive values indicate target overshoot. Error bars reflect standard error of the mean. ${ }^{*} p<.05$. 


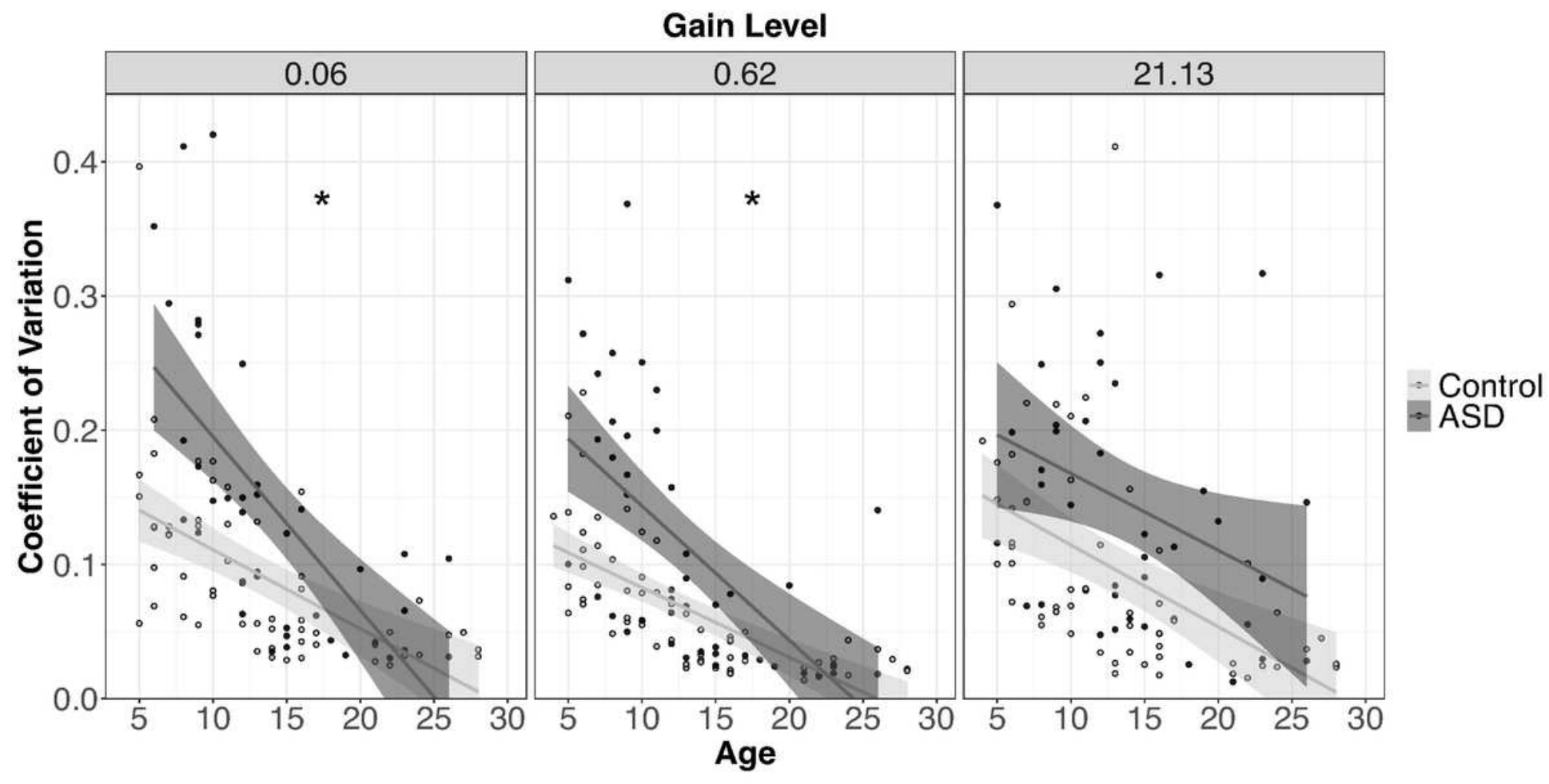

Figure 6

Variability of force output varies as a function of age and amount of visual feedback. Individuals with ASD demonstrated greater age-associated reductions in force variability (coefficient of variation) than control participants at low and medium gain. Age-related reductions in force variability were similar in ASD and controls at high gain, but overall variability was increased in ASD participants. Error bars reflect standard error of the mean. *slopes differ between groups. 


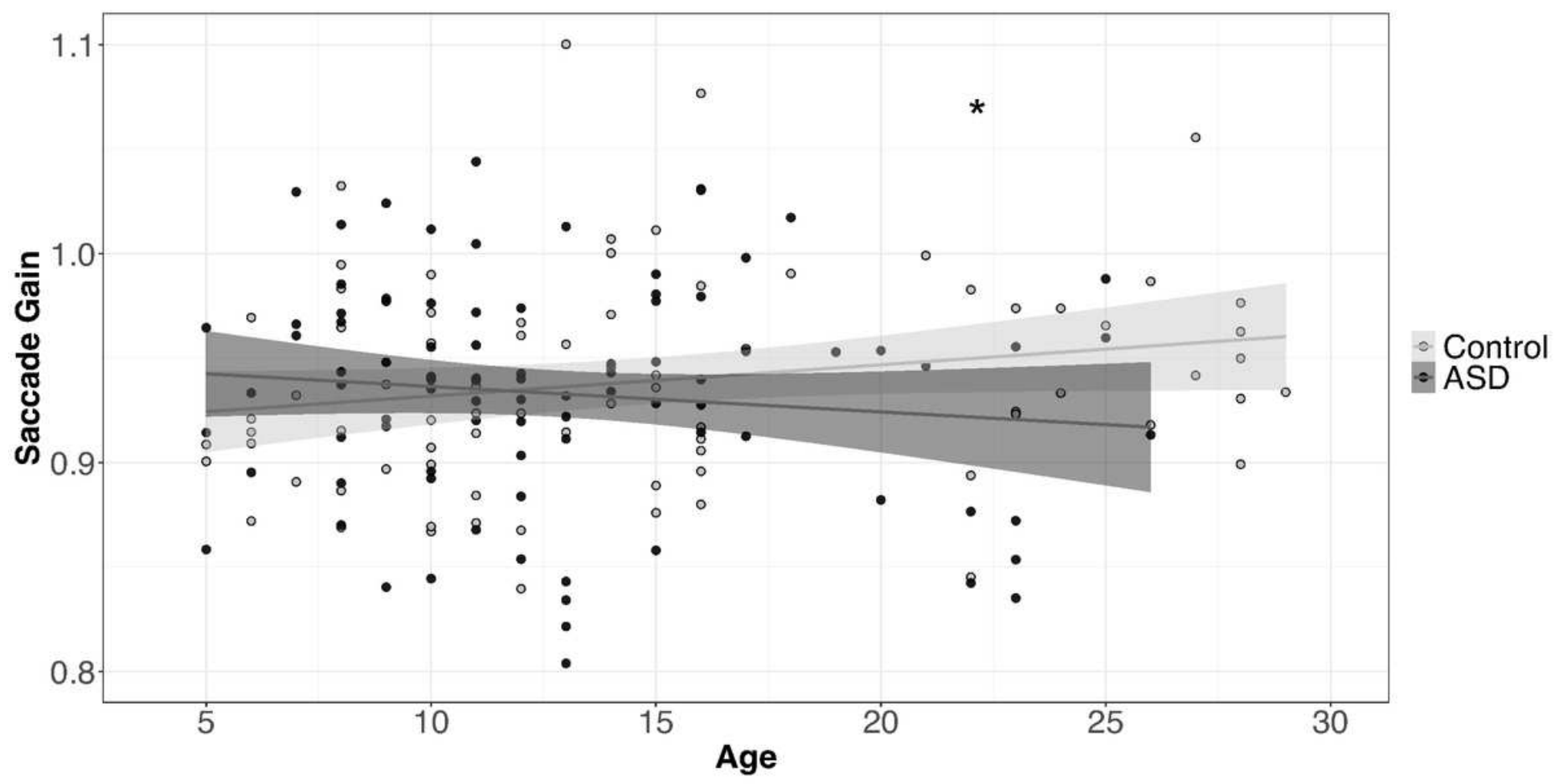

Figure 7

Increased age was associated with increased saccade accuracy in controls and with more severe hypometria in individuals with ASD. Error bars reflect standard error of the mean. *slopes differ between groups. 


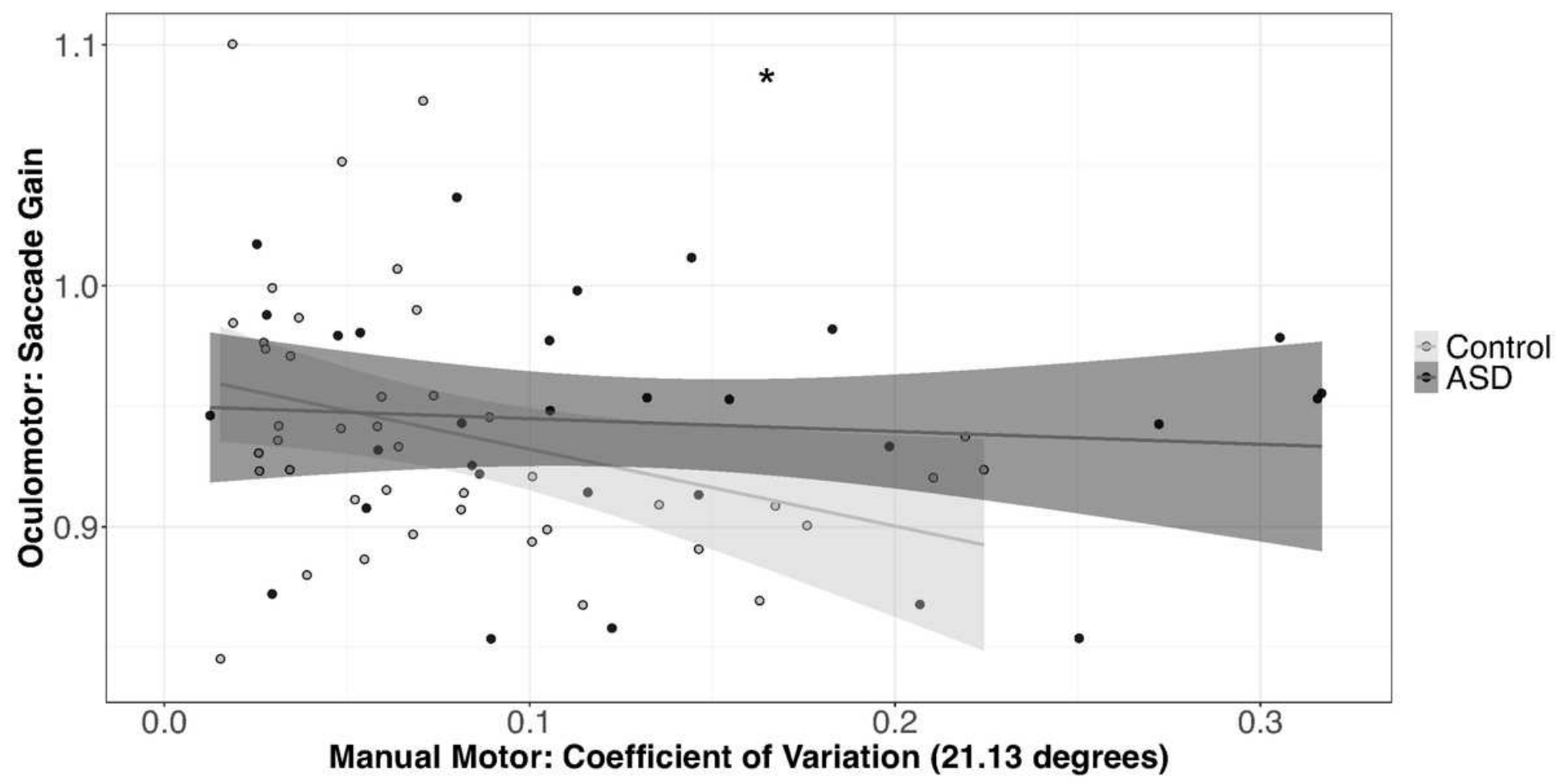

Figure 8

Increased saccade accuracy was associated with reduced CoV in controls, but not in individuals with ASD, across multiple task conditions (Gain task: 21.13 degrees depicted). Error bars reflect standard error of the mean. *slopes differ between groups.

\section{Supplementary Files}

This is a list of supplementary files associated with this preprint. Click to download.

- SupplementaryFigures.docx 